\title{
Les occupations mésolithiques de Bourrouilla à Arancou (Pyrénées-Atlantiques, France)
}

Mesolithic occupations in Bourrouilla cave at Arancou (Pyrénées-Atlantiques, France)

Morgane Dachary, Jean-Claude Merlet, Mathilde Miquéou, Jean-Baptiste Mallye, Olivier Le Gall et Anne Eastham

\section{OpenEdition}

\section{Journals}

Édition électronique

URL : http://journals.openedition.org/paleo/2543

DOI : $10.4000 /$ paleo.2543

ISSN : 2101-0420

Éditeur

SAMRA

\section{Édition imprimée}

Date de publication : 15 décembre 2013

Pagination : $79-102$

ISSN : $1145-3370$

Référence électronique

Morgane Dachary, Jean-Claude Merlet, Mathilde Miquéou, Jean-Baptiste Mallye, Olivier Le Gall et Anne Eastham, « Les occupations mésolithiques de Bourrouilla à Arancou (Pyrénées-Atlantiques, France) », PALEO [En ligne], 24 | 2013, mis en ligne le 17 avril 2014, consulté le 07 juillet 2020. URL : http:// journals.openedition.org/paleo/2543; DOI : https://doi.org/10.4000/paleo.2543

Ce document a été généré automatiquement le 7 juillet 2020

\section{c) (1) $\odot \odot$}

PALEO est mis à disposition selon les termes de la licence Creative Commons Attribution - Pas d'Utilisation Commerciale - Pas de Modification 4.0 International. 


\title{
Les occupations mésolithiques de Bourrouilla à Arancou (Pyrénées- Atlantiques, France)
}

\author{
Mesolithic occupations in Bourrouilla cave at Arancou (Pyrénées-Atlantiques, \\ France) \\ Morgane Dachary, Jean-Claude Merlet, Mathilde Miquéou, Jean-Baptiste \\ Mallye, Olivier Le Gall et Anne Eastham
}

\section{Introduction (MD)}

1 Le Mésolithique de l'extrême sud-ouest de la France est aujourd'hui encore mal connu. En raison de fouilles anciennes (impliquant une absence de tamisage ou un tamisage insuffisant), parce que les séquences sont perturbées ou que les séries sont numériquement très faibles, peu de sites ont fourni des données fiables sur les derniers chasseurs-cueilleurs entre le sud des Landes et la frontière espagnole.

Dans ce contexte régional, la découverte, dans la grotte de Bourrouilla à Arancou (Pyrénées-Atlantiques), de témoins d'occupations mésolithiques apporte de précieuses données. La conservation de la faune, qui fait cruellement défaut dans les sites de pleinair d'Aquitaine, et la réalisation de deux datations radiocarbone constituent, en plus des vestiges lithiques, des éléments très utiles à une meilleure compréhension du Mésolithique du versant nord de l'extrémité occidentale des Pyrénées.

La grotte de Bourrouilla est située dans les Pyrénées-Atlantiques, sur la commune d'Arancou (fig. 1). La découverte du gisement a eu lieu en 1986, en même temps qu'une fouille clandestine qui affectait l'intérieur de la cavité. Après le tamisage et le tri des déblais de la fouille clandestine qui a mis en avant le potentiel et la richesse du site, puis la réalisation d'un sondage extérieur visant à cerner l'extension des occupations et la puissance de la stratigraphie (Chauchat et al. 1999), des fouilles programmées ont été 
entreprises dès 1998, d'abord sous la responsabilité de Cl. Chauchat, puis sous celle de M. Dachary.

Figure 1 - Carte de situation de Bourrouilla et des principaux sites mésolithiques cités.

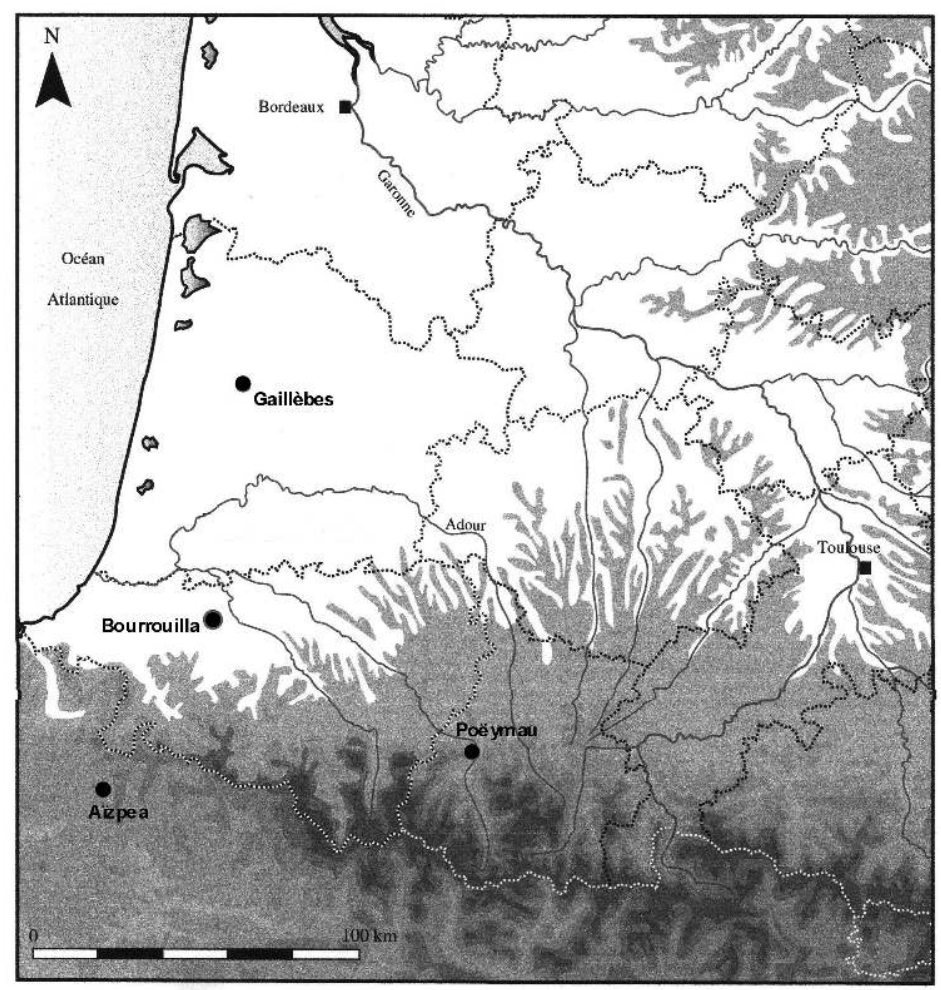

De manière à mieux situer le gisement dans son environnement, notons que la grotte s'ouvre à $50 \mathrm{~m}$ d'altitude, dans les calcaires à Mélobésiées de l'Albien. Elle domine la rive droite du ruisseau du Lauhirasse, un petit affluent de la Bidouze, elle-même tributaire des Gaves réunis.

L'analyse du contexte géographique montre que plusieurs éléments présents dans l'environnement immédiat de la grotte ont pu favoriser l'installation de l'Homme :

- la présence de l'eau (Dachary 1993) en tant qu'élément indispensable à la vie (petites sources à quelques dizaines de mètres) mais aussi en tant que potentiel de ressources alimentaires aussi bien pour la Bidouze que pour le Gave d'Oloron (Le Gall in Chauchat et al. 1999) ;

- l'existence au Mésolithique d'au moins deux milieux écologiques terrestres que sont les collines boisées du piémont et les zones de marais proches des cours d'eau, dans un rayon de $2 \mathrm{~km}$ environ (Eastham in Chauchat et al. 1999) ;

- une situation topographique idéale : une ouverture orientée au sud-est - donc à l'abri des vents dominants - et située sur un plateau surplombant les torrents.

Le gisement est classiquement subdivisé en quatre secteurs (fig. 2) : la zone extérieure (bandes 25 à 28), le vestibule à l'aplomb du porche (bandes 22 à 24), le couloir (bandes 19 à 21) et la salle du fond (bandes 15 à 18). Bien que l'essentiel de la stratigraphie documente des occupations magdaléniennes, des témoins d'occupations mésolithiques ont été découverts dans la zone extérieure, le vestibule et la salle du fond. 
Figure 2 - Plan du gisement de Bourrouilla et localisation des locus ayant livré les vestiges mésolithiques.

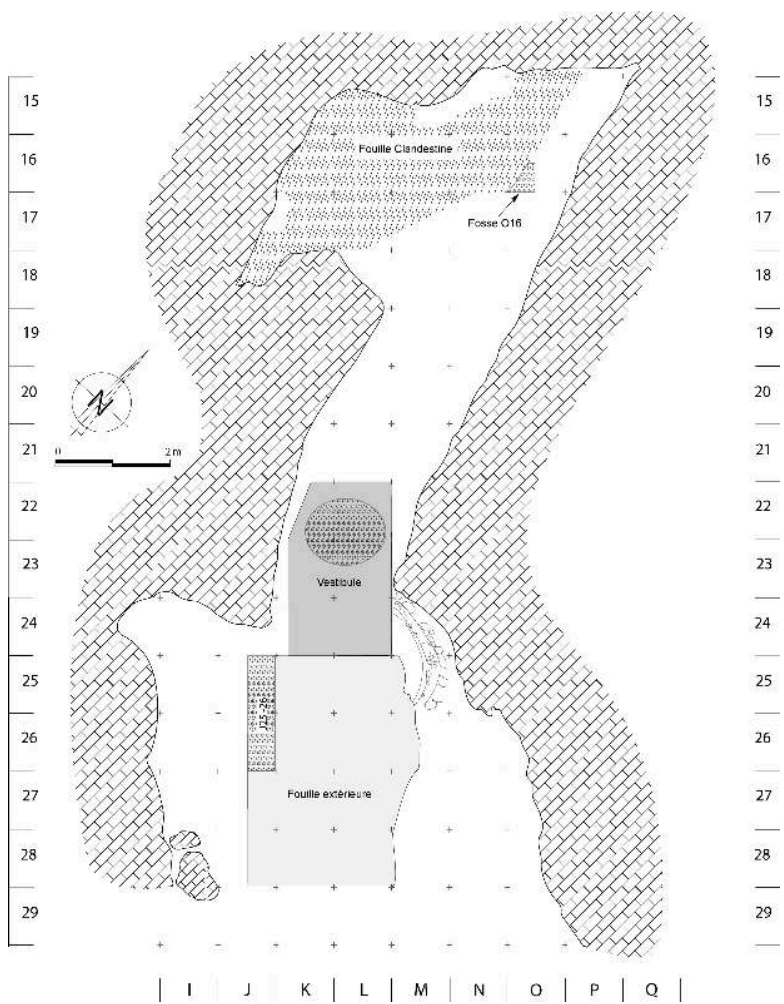

7 Les indices mésolithiques forment trois ensembles, issus des trois locus mentionnés supra, et offrent des états de conservation différents. A l'extérieur, la fouille des carrés $\mathrm{J} 25$ et J26 a fourni une série homogène au sein d'une stratigraphie nette. Ce secteur, bien conservé, a fait l'objet d'une fouille fine par horizons artificiels de $2 \mathrm{~cm}$ d'épaisseur, avec tamisage à l'eau des sédiments recueillis par quart de mètre carré. Dans le vestibule, un ensemble sédimentaire fortement bioturbé (notamment par l'action de blaireaux) a fait l'objet d'un prélèvement en masse, sans cotation du matériel. Les sédiments ont été tamisés à l'eau avec un tamis à maille fine, puis triés. Les vestiges lithiques extraits ont alors bénéficié d'un traitement destiné à dissoudre les concrétions plus ou moins épaisses qui les enrobaient. L'outillage a ensuite été isolé. En d'autres termes, une partie de la fraction fine est absente, sans doute parce que, concrétionnée, elle n'a pas été repérée au cours du tri.

Enfin, un troisième lot de vestiges provient de la fouille partielle d'une fosse (US 2002) creusée dans les niveaux magdaléniens de la salle du fond (carré 016). En bordure de la fouille clandestine, cette structure en creux, visible dans la coupe témoin, menaçait de s'effondrer. Elle a donc été fouillée par horizons artificiels de $2 \mathrm{~cm}$, sur un peu moins d'un quart de mètre carré. Les sédiments ont été tamisés à l'eau avec un tamis à maille fine, puis triés.

9 Dans cet article, nous présenterons le matériel par catégories de vestiges et par secteur avant de discuter de la valeur de la série puis de la replacer dans un contexte plus large, à l'échelle du Mésolithique du sud-ouest de la France. 


\section{1 - L'industrie lithique (JCM et MM)}

\section{1 - Présentation générale de l'industrie}

10 L'industrie lithique est présente dans les trois secteurs contenant des niveaux mésolithiques : bande J (à l'extérieur), vestibule, et fosse 016 (dans la salle du fond). Il apparaît nécessaire d'aborder séparément chacun de ces secteurs, pour tenir compte de la variabilité de leur mode de dépôt et de conservation, avant d'avoir une vision globale des fréquentations mésolithiques du site.

11 L'idéal serait de distinguer des ensembles technologiquement significatifs, permettant de décrire des phasages. Mais l'exercice est impossible ici, en raison de l'inégalité quantitative et qualitative des trois séries et du contexte de la fouille.

Le débat étant toujours d'actualité en Aquitaine sur les modalités et les implications des évolutions techniques au cours des différentes phases du Mésolithique (Valdeyron 2000 ; Roussot-Larroque 2005, 2009), nous avons choisi de nous référer à la division entre un Premier Mésolithique (phases ancienne et moyenne) et un Second Mésolithique (phases récente et finale).

- Le Premier Mésolithique correspond aux industries à triangles, lamelles étroites à bord abattu, supports étroits obtenus par percussion directe tendre minérale. Dominées par les triangles isocèles et les hypermicrolithes effilés, ces industries sont représentées dans le sud-ouest de la France par le Sauveterrien ancien et moyen.

- Le Second Mésolithique correspond aux industries à trapèzes et armatures larges, supports larges, réguliers et standardisés, avec apparition de la percussion indirecte.

13 L'accent sera mis sur quelques traits paraissant pertinents, en s'attachant notamment aux armatures microlithiques, car ces pièces, fortement investies au plan technoculturel, sont les éléments les plus diagnostiques de l'ensemble.

Etant donné la nature très variée des typologies existantes, nous devons aussi préciser la définition de certains termes employés. La terminologie adoptée reprend des éléments de la typologie du GEEM (GEEM 1969, 1972, 1975), révisée par Grégor Marchand (Marchand 1999). Nous avons distingué trois familles au sein des pièces retouchées :

- Famille des armatures géométriques (groupes des bitroncatures et des troncatures/bords abattus).

- Famille des armatures non géométriques (groupes des troncatures/bords abattus et des bords abattus).

- Famille des outils du fonds commun (groupes des bords abattus, des lames et lamelles à coches, des troncatures, des grattoirs, des perçoirs, des pièces retouchées, des outils a posteriori ou pièces usées).

15 Précisons également que nous avons distingué lames, lamelles et micro-lamelles, sur des critères morphométriques (rapport longueur/largeur, largeur de lamelle $<12 \mathrm{~mm}$ ).

\subsection{1 - Décompte du mobilier lithique}

L'ensemble du mobilier lithique des niveaux mésolithiques d'Arancou compte 9915 pièces en silex, dont 185 retouchées, 213 lames/lamelles et 16 nucléus. Leur répartition par grandes catégories et par locus est détaillée dans le tableau 1. 
17 Les grandes lignes de l'analyse technologique sont présentées ci-après; les résultats détaillés sont exposés dans les rapports de fouilles de 2010 et 2012 (Merlet et Miquéou in Dachary et al. 2010, 2012). L'étude a porté plus précisément sur les pièces diagnostiques (armatures, pièces techniques, nucléus) qui représentent 238 éléments (tabl. 2), puis sur les lames et lamelles. Le reste du mobilier a été étudié en fonction des observations réalisées sur les pièces diagnostiques.

\subsection{2 - L'approvisionnement en matériaux siliceux}

18 Peu de pièces autorisent une détermination du silex utilisé, car beaucoup présentent une altération (patine, chauffe) ou sont de dimensions trop réduites. L'examen de celles qui peuvent être déterminées montre la présence quasi-exclusive du silex du Flysch "de type Bidache». La grotte de Bourrouilla est comprise dans la zone du Flysch crétacé du piémont pyrénéen, et ce matériau est ici accessible dans un rayon de quelques kilomètres, où il affleure sous divers faciès. Il se présente généralement sous forme de plaquettes épaisses à structure litée et à texture le plus souvent grainstone. Plusieurs armatures sont confectionnées dans ce matériau, tout comme les nucléus.

Tableau 1 - Composition de l'industrie lithique mésolithique de la grotte Bourrouilla.

\begin{tabular}{|c|c|c|c|c|}
\hline Lithique & $\begin{array}{c}\text { vestibule } \\
\text { remanié }\end{array}$ & J25-J26 & $\begin{array}{c}016 \\
\text { (US 2002) } \\
\end{array}$ & Total \\
\hline lames/lamelles & 144 & 14 & 55 & 213 \\
\hline éclats silex & \multirow{2}{*}{7336} & 68 & 322 & \multirow{2}{*}{9418} \\
\hline fragments silex & & 245 & 1447 & \\
\hline cupules thermiques & 0 & 17 & 29 & 46 \\
\hline nucléus & 9 & 0 & 7 & 16 \\
\hline blocs testés & 2 & 0 & 0 & 2 \\
\hline pièces techniques & 30 & 0 & 5 & 35 \\
\hline pièces retouchées & 137 & 19 & 29 & 185 \\
\hline Total silex & 7658 & 363 & 1894 & 9915 \\
\hline $\begin{array}{l}\text { autres fragments lithiques } \\
\text { (grès, cristal de roche, quartzite) }\end{array}$ & $\begin{array}{c}\text { non } \\
\text { décomptés }\end{array}$ & 4 & 172 & 176 \\
\hline
\end{tabular}

On note l'absence de silex allochtones, tels que le silex à Lépidorbitoïdes dont on connaît de nombreux gîtes en Chalosse ou encore les petits galets de module inférieur à $10 \mathrm{~cm}$ et à cortex d'érosion marine disponibles sur le littoral atlantique. L'approvisionnement ici s'est donc effectué dans un périmètre de quelques kilomètres autour de la grotte.

Il ne faudrait pas en conclure hâtivement que les groupes ayant fréquenté la cavité ne se déplaçaient qu'au sein d'un territoire restreint. Les travaux récents montrent que, plus au nord dans les Landes, les Mésolithiques de Gaillèbes-2 s'approvisionnaient sur le littoral et en Chalosse, sources distantes de $45 \mathrm{~km}$ (Merlet et Miquéou 2009). Sur le versant sud des Pyrénées, dans les niveaux inférieurs d'Aizpea (datés de $7790 \pm 70$ et $7170 \pm 70 \mathrm{BP}$ ), un petit pourcentage de silex provient de Chalosse et des galets dont le cortex a subi une abrasion marine "de la zone littorale des Landes ", ainsi que des silex du Flysch pourraient aussi venir du Pays Basque nord (Cava et al. 2007-2008), impliquant des déplacements de plus de 50 kilomètres. A Bourrouilla, ils pouvaient en réalité exploiter les ressources d'un espace de vie assez large comportant différentes 
haltes et impliquant des mouvements sur des distances, là aussi, de plusieurs dizaines de kilomètres. Simplement, les pièces abandonnées ici reflètent la confection de l'équipement lithique nécessaire aux activités développées sur place à partir d'un choix opportuniste des matériaux dont ils disposaient à proximité. En d'autres termes, le silex du Flysch répondait à leurs exigences et à leurs habitudes.

Tableau 2 - Répartition des pièces diagnostiques de la série mésolithique de la grotte Bourrouilla.

\begin{tabular}{|c|c|c|c|c|}
\hline Pièces diagnostiques & $\begin{array}{c}\text { Vestibule } \\
\text { remanié }\end{array}$ & $\begin{array}{c}\text { J25-J26 } \\
\text { tamisage }\end{array}$ & $\begin{array}{c}\text { O16 } \\
\text { (US 2002) }\end{array}$ & Total \\
\hline armatures géométriques & 21 & 4 & 6 & 31 \\
\hline $\begin{array}{c}\text { armatures non } \\
\text { géométriques }\end{array}$ & 74 & 5 & 9 & 88 \\
\hline outils du fonds commun & 16 & 2 & 5 & 23 \\
\hline Indéterminables & 21 & 7 & 8 & 36 \\
\hline microburins & 5 & 1 & 1 & 7 \\
\hline nucléus & 9 & 0 & 7 & 16 \\
\hline blocs testés & 2 & 0 & 0 & 2 \\
\hline pièces techniques & 30 & 0 & 5 & 35 \\
\hline $\begin{array}{c}\text { Sous-total pièces } \\
\text { retouchées }\end{array}$ & 137 & 19 & 29 & 185 \\
\hline Sous-total débitage & 41 & 0 & 12 & 53 \\
\hline Total & 178 & 19 & 41 & 238 \\
\hline
\end{tabular}

\section{2 - Les indices d'occupation à l'extérieur}

\section{(J25 et J26)}

L'industrie lithique recueillie en J25 et J26 est numériquement faible (363 pièces) et peu caractéristique. On ne compte aucun nucléus, seulement huit lames, six lamelles, 19 pièces retouchées et 334 autres produits de débitage. Les 19 pièces retouchées comportent neuf armatures dont quatre géométriques : une bipointe aux deux bords abattus apparentée à une pointe de Sauveterre, un triangle isocèle et deux triangles scalènes dont le troisième côté est retouché. Les supports lamino-lamellaires montrent l'usage de la percussion directe tendre. Les profils sont principalement rectilignes et les nervures sinueuses. Les autres pièces comprennent surtout des fragments $(74,5 \%)$ et éclats de silex (19,8\%), ainsi que des esquilles et des cupules thermiques. Ils se répartissent en trois grandes classes typométriques avec $46,3 \%$ de dimension maximum inférieure à $5 \mathrm{~mm}, 39,9 \%$ de dimension entre 5 et $10 \mathrm{~mm}$ et seulement $13,8 \%$ de pièce de plus de $10 \mathrm{~mm}$. Plus de $60 \%$ des produits présentent des traces de chauffe.

Pour ce secteur, au regard de l'association " pointe de Sauveterre - triangle isocèle scalènes avec retouche des trois côtés", on peut envisager la présence d'un Premier Mésolithique. Il n'y a pas de contamination apparente par des niveaux sous-jacents. La 
forte proportion de pièces fragmentées, brûlées, et de produits de débitage $\leq 5 \mathrm{~mm}$, invite à voir cet espace comme une zone de rejet.

Figure 3 - Vestibule. Armatures géométriques et non géométriques. Premier Mésolithique.

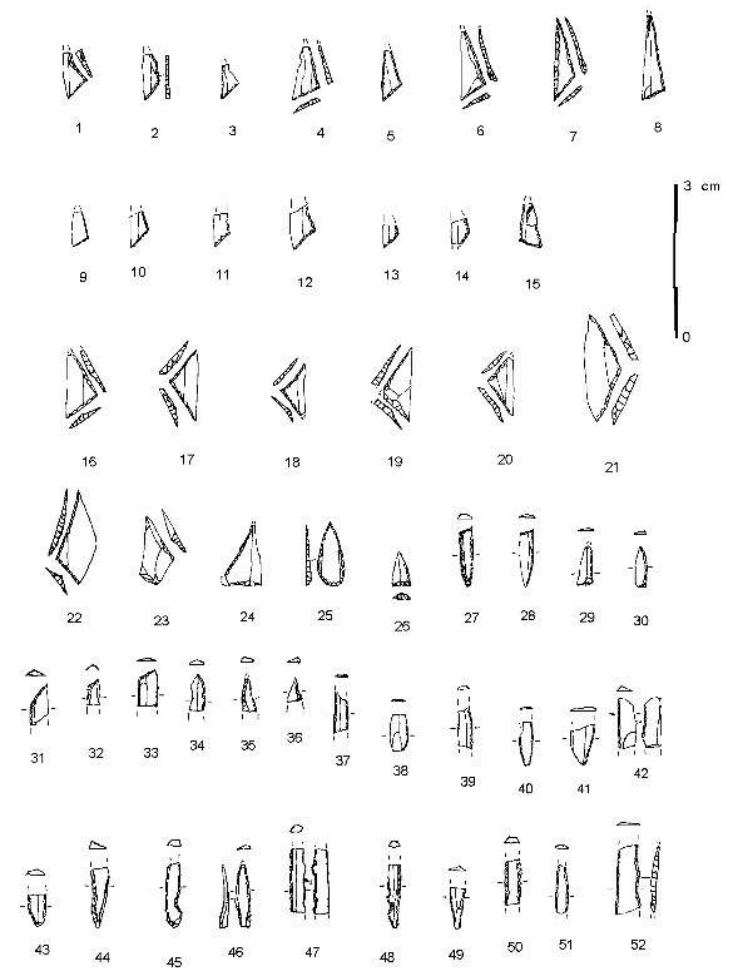

1 à 16, 19 : Triangles scalènes. 17, 18, 20, 21 : Triangles isocèles. 22 : Pointe par bitroncature. 23 : Pointe à base naturelle. 24 : Trapèze asymétrique. 25 : Pointe à base convexe. 26 : Pointe à base concave. 27 et 28 : Lamelles scalènes. 29, 30, 34 à 52 : Lamelles à bord abattu. 31 à 33 : Lamelles tronquées (@ J.-C. Merlet).

\section{3 - Les indices d'occupation du vestibule}

La série du vestibule est numériquement la plus importante : 7658 silex. Elle comprend neuf nucléus et deux blocs testés, 31 lames, 107 lamelles, 137 pièces retouchées et 7366 autres produits de débitage. On sait cependant que l'ensemble sédimentaire dont elle est issue est remanié. Il convient donc d'abord d'écarter, en s'appuyant sur des critères morpho-techniques, les pièces attribuables avec assez de sûreté au Magdalénien et/ou à l'Epipaléolithique. Tel est le cas des cinq burins et 21 chutes, des quatre pointes larges à bord abattu, d'une partie des lames, etc. Le problème est plus complexe pour les lamelles à dos qui peuvent se rencontrer dans les industries mésolithiques et sont donc difficiles à rattacher à l'une ou l'autre de ces périodes.

\subsection{1 - Les pièces retouchées}

Les armatures géométriques comprennent 21 triangles (17 scalènes et quatre isocèles) et deux lamelles scalènes (fig. 3). Plusieurs triangles ordinaires courts et une lamelle scalène présentent un caractère évolué: le troisième côté retouché. Les supports sont principalement des lamelles étroites comme l'attestent les nervures visibles et le gabarit, ces triangles ne dépassant pas les $7 \mathrm{~mm}$ de large et les $2 \mathrm{~mm}$ 
d'épaisseur. La mesure de l'angle que forment les deux troncatures, en permettant d'aborder les pièces non entières, vient confirmer le classement effectué, plusieurs de ces pièces portant également une retouche du troisième côté, observation qui se corrèle parfaitement avec ce qui précède. Une pièce rappelle la forme du segment. Un trapèze asymétrique sur lamelle à deux pans possède des bords et nervures rectilignes (fig. 3, $\mathrm{n}^{\circ}$ 24). Les retouches directes semi-abruptes forment deux troncatures: une troncature oblique et une troncature droite. Enfin, il y a une flèche de Montclus (fig. 4, $\mathrm{n}^{\circ} 37$ ), mesurant $9,25 \mathrm{~mm}$ de large et 2,11 $\mathrm{mm}$ d'épaisseur.

Figure 4 - Vestibule. Armatures non géométriques, microburins, divers.

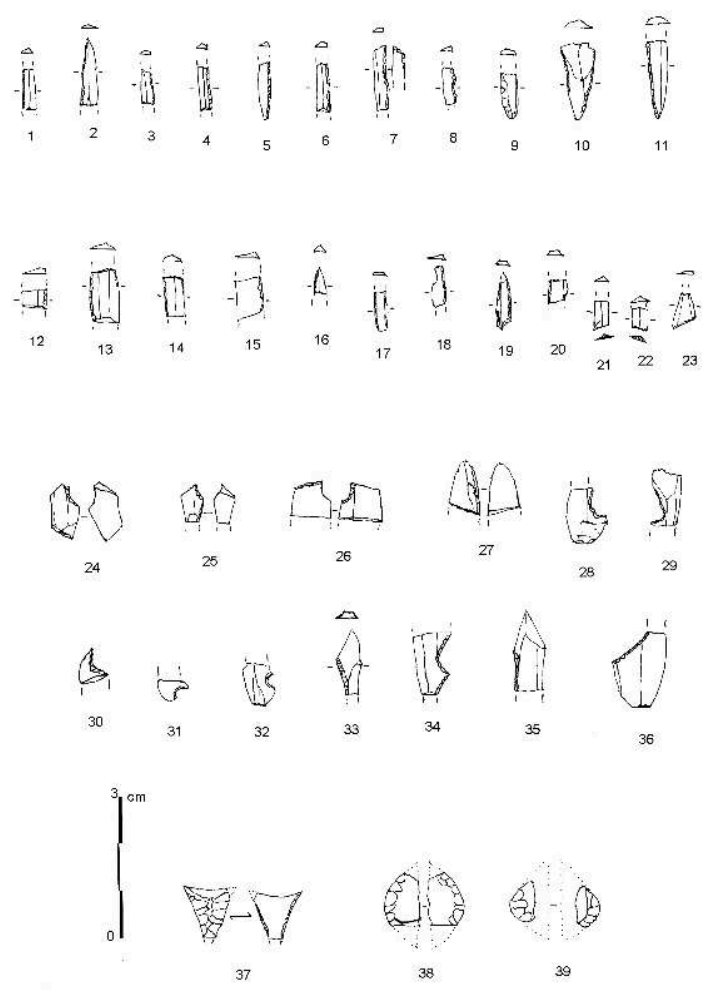

1 à 20 : lamelles à dos. 21 et 22 : lamelles à base tronquée.

23 : lamelle à base retouchée. 24 et 25 : micro-burins. 26 à 35 : déchets de façonnage des armatures lamelles à coches, lamelles à troncature partielle. 36 : troncature oblique. 37 : flèche de Montclus. 38 et 39 : segments du Bétey (37 à 39 : Second Mésolithique/ Néolithique ancien) (@ J.-C. Merlet).

Les pointes. On dénombre 15 pointes (fig. 3 et 4) réparties comme suit : trois pointes étroites à un bord abattu, quatre pointes étroites à deux bords abattus, quatre pointes sur troncature oblique et quatre pointes larges à un bord abattu (sur lame). Les indices d'allongement indiquent deux groupes: un sur supports longs et larges, l'autre sur supports plus courts et étroits. De plus, lorsque les nervures sont apparentes, elles sont sinueuses. Les armatures les plus fragmentées sont les pointes étroites. Une seulement est entière. Pour les autres, une seule des extrémités a été fracturée. L'angle des extrémités perçantes des pointes étroites à bords abattus et des pointes sur troncature oblique oscille entre $40^{\circ}$ et $55^{\circ}$, ce qui vient appuyer l'interprétation d'un emmanchement en pointe axiale (Domingo Martinez 2005 ; Chesnaux 2008) même si les fractures observées sur l'apex ne portent pas de stigmates d'impact (Fischer, Wemming-Hansen, Rasmussen 1984). Les pointes étroites à un bord abattu sont à retouches directes abruptes écailleuses tandis que celles à deux bords abattus sont 
réalisées par retouches directes semi-abruptes écailleuses et envahissantes. Il y a une pointe étroite à un bord abattu et une pointe étroite à deux bords abattus avec une base en partie proximale.

Les lames et lamelles à bords abattus. Ce groupe comprend 54 pièces dont deux lames à deux bords abattus. Les lamelles (fig. 4) peuvent être divisées en quatre types :

- 30 lamelles à un bord abattu à retouches directes,

- 1 lamelle à un bord abattu à retouches inverses,

- 15 lamelles à deux bords abattus à retouches directes,

- 6 lamelles à deux bords abattus à retouches alternes.

Seules trois armatures sont entières parmi les lamelles. Pour les autres, ce sont les parties mésiales puis méso-distales qui ont été majoritairement conservées. Nous avons un ensemble de lamelles homogènes du point de vue des dimensions, mais variables du point de vue des retouches. Les bords sont rectilignes et la retouche est continue sur tout le bord.

Les lames et lamelles tronquées. Outre une lame tronquée à deux bords abattus, l'effectif comprend surtout neuf lamelles tronquées (fig. 3, n 31 à 33) dont quatre à un bord abattu, trois à deux bords abattus et deux lamelles tronquées simples. Le gabarit du support retouché est assez constant, au sein de deux catégories : lamelles et microlamelles. Lorsque les nervures sont apparentes, elles sont sinueuses. Les retouches sont directes semi-abruptes à abruptes écailleuses et elles portent sur la totalité des bords. Les mesures des angles formés par l'intersection d'un bord abattu avec la troncature montrent pour deux lamelles tronquées à un bord abattu des angles de $100^{\circ}$ et $110^{\circ}$. Nous pourrions parfaitement les intégrer aux lamelles scalènes.

Lames et lamelles à coches. Les lamelles à coches (fig. 4) sont toutes fracturées au niveau de la coche après façonnage. Il pourrait donc s'agir de déchet de fabrication d'armatures, provenant d'un fractionnement des supports et non de lamelles à coches stricto sensu. D'autant que seule la coche est retouchée (les bords ne sont pas retouchés), à l'exception de deux lamelles à bord abattu et coche sur le même bord. Les largeurs varient entre 5,5 et 7,5 $\mathrm{mm}$ d'une part et 8,5 et $9 \mathrm{~mm}$ d'autre part. Les épaisseurs sont inférieures à $2 \mathrm{~mm}$. Toutes les retouches sont directes, courtes, semiabruptes et écailleuses.

Les outils du fonds commun comprennent deux grattoirs, un perçoir, une troncature et deux denticulés.

31 Autres pièces retouchées, indéterminables. 21 pièces, parmi lesquelles on peut identifier six fragments d'armatures.

Les pièces techniques comptent 22 chutes de burin et trois crêtes d'entretien. La présence concomitante de chutes de burin et de cinq burins, indique manifestement une intrusion de mobilier magdalénien.

Les microburins (fig. 4, nos 24, 25) sont au nombre de cinq. Bien que recueillis dans le contexte remanié du vestibule, ils peuvent être rattachés par leur morphologie au Premier Mésolithique.

\subsection{2 - Les produits de débitage}

Nucléus. On décompte neuf nucléus entiers et deux blocs testés. L'un des nucléus a été réalisé à partir d'un éclat épais, la face d'éclatement ayant servi de plan de frappe. Il n'y 
a qu'une seule table de débitage. Le débitage est semi-tournant et les négatifs des derniers enlèvements sont allongés (lames/lamelles ?). Les huit autres nucléus ont été réalisés sur ce qui semblait être des galets mais seuls deux exemplaires conservent du cortex. Celui-ci est granuleux et vacuolaire. Concernant le volume final, les nucléus unipolaires sont de petite dimension, ne dépassant pas les $30 \mathrm{~mm}$ de haut. Ils sont très semblables dans la mesure où les négatifs d'enlèvements finaux sont des éclats, mis à part pour deux d'entre eux. Les plans de frappe sont lisses et associés à une table de débitage qui se prolonge sur les côtés du nucléus pour l'essentiel. Les nucléus bipolaires mesurent entre 34 et $65 \mathrm{~mm}$ de haut tandis que les blocs testés mesurent entre 68 et 76 mm de haut.

Supports lamino-lamellaires. Les 144 supports lamino-lamellaires (fig. 5), comprennent 37 lames et 107 lamelles. Seulement $16,7 \%$ sont entiers pour $61,8 \%$ de fragments mésio-proximaux, $10,4 \%$ de fragments mésio-distaux et $11,1 \%$ de fragments mésiaux. Néanmoins, il a été possible d'observer suffisamment de talons pour avoir un aperçu de la technique de percussion. Ainsi, les talons sont-ils souvent inframillimétriques ou lisses avec une forte abrasion de la corniche, voire un doucissage. Les angles entre le bord du plan de frappe et la table de débitage oscillent entre $70^{\circ}$ et $80^{\circ}$. Les bulbes sont très peu volumineux. Il semble donc que la percussion directe tendre soit dominante. Certaines pièces présentent des indices plus caractéristiques qui permettent de préciser le type de percussion. En effet, le cône de percussion est parfois nettement marqué avec des étoilures, des fissurations, des esquillements du bulbe et parfois même des points de contact multiples. La percussion directe tendre minérale est alors avérée. Sur l'ensemble de ces produits nous n'avons pu repérer de percussion indirecte.

Figure 5 - Vestibule. La production lamino-lamellaire.

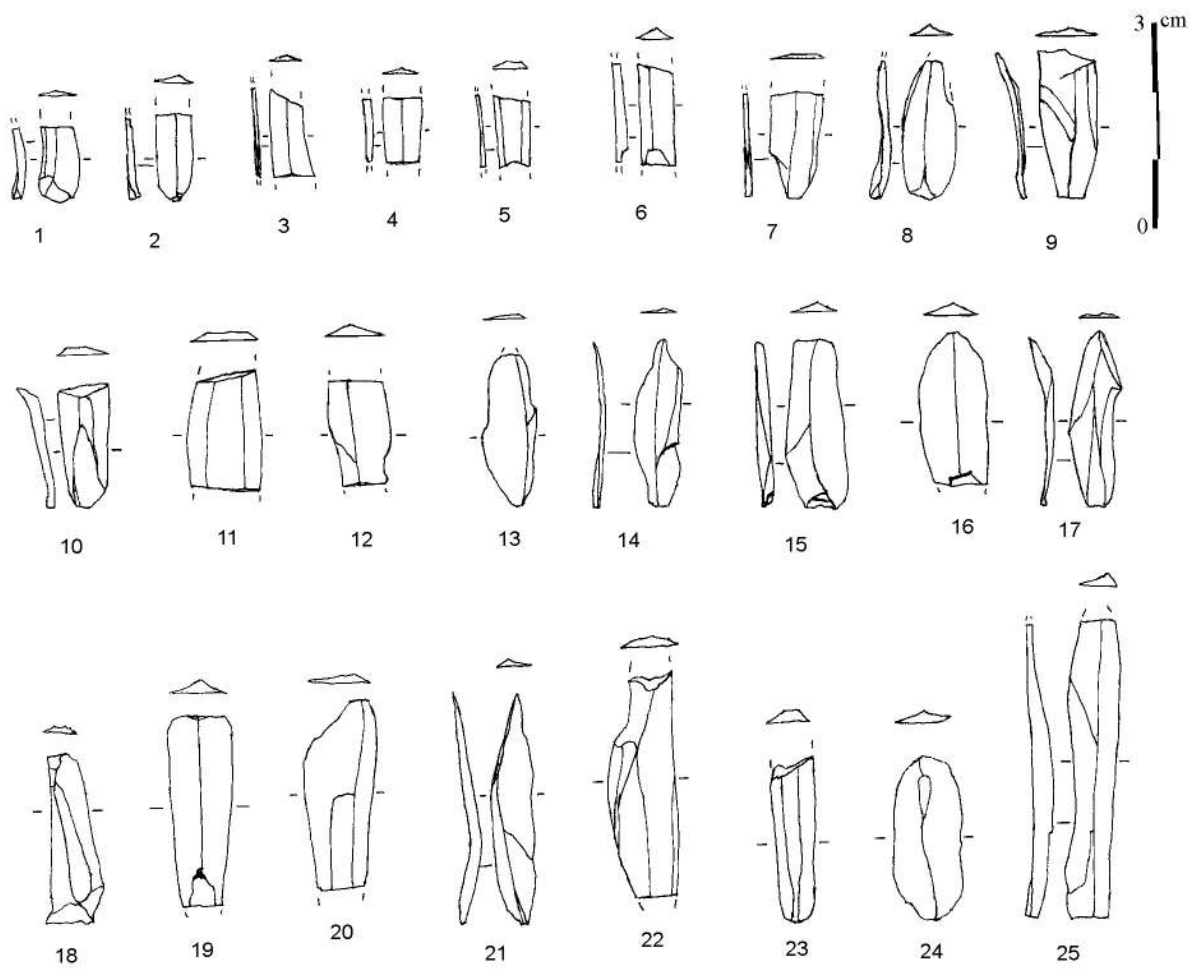

1 à 25 : petites lames et lamelles brutes (@ J.-C. Merlet). 
36

Eclats, esquilles, fragments. On dénombre 7336 éclats, fragments et cupules, dans le vestibule, se répartissant à parts sensiblement égales entre pièces de dimension inférieure à $1 \mathrm{~cm}$ et pièces dépassant $1 \mathrm{~cm}$. Le tri a été fait avant traitement des concrétions calcaires, ce qui peut expliquer la sous-représentation de la fraction très fine.

Figure 6 - Industrie lithique de la fosse 016.

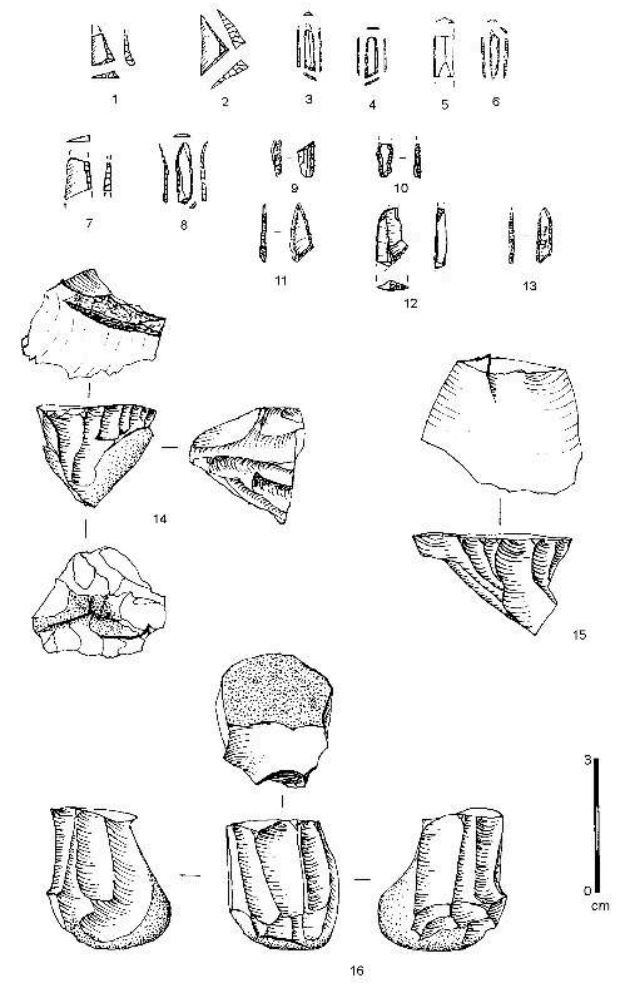

$1,3,4,11,12$ : triangles scalènes. 2 : triangle isocèle. 5 et $10:$ lamelles à bord abattu. 6 : pointe sur troncature oblique. 7 et 9 : fragments de microlithes. 8 et 13 : pointes à bords abattus, assimilables à des pointes de Sauveterre.

14 à 16 : nucléus en silex du Flysch ( 1 à 8 ,

14 à 16 : (c) J.-C. Merlet; 9 à 13 : (c) M. Dachary).

\section{4 - Les indices d'occupation dans la salle du fond : 016 (US 2002)}

Cette série, quoique plus restreinte, est homogène. Elle est donc la plus apte à fournir des informations de qualité. Elle compte 1894 produits dont sept nucléus, 15 lames, 40 lamelles, 29 pièces retouchées et 1803 autres produits de débitage.

Les armatures. Elles comportent cinq triangles scalènes et un isocèle (fig. 6). Les supports semblent être des lamelles étroites comme l'attestent les nervures visibles et le gabarit. Les caractères évolués dominent dans la mesure où quatre triangles ont les trois côtés retouchés et ont une tendance à l'allongement comme l'indiquent l'indice d'allongement, l'indice de symétrie, ainsi que l'angle formé par les troncatures. On dénombre trois pointes dont deux sont assimilables à des pointes de Sauveterre, et une pointe à troncature oblique. Elles présentent les mêmes caractéristiques que celles rencontrées dans le vestibule. 
Les dix lames et lamelles à bords abattus peuvent être divisées en trois types : quatre lames à un bord abattu, cinq lamelles à un bord abattu à retouches directes, une lamelle à deux bords abattus à retouches directes. Aucune n'est entière. Ce sont les parties mésiales et mésio-proximales qui ont été conservées. Il y a quasiment autant de bords abattus abrupts que de bords semi-abrupts. Ainsi, seul le gabarit des pièces fait varier cet ensemble pour le reste très homogène.

L'outillage du fonds commun est des plus restreints : un racloir et huit éléments retouchés indéterminés, pour la plupart sur éclat.

Parmi les sept nucléus (fig. 6, nos 14 à 16), six sont entiers et un fragmentaire. En silex du Flysch, ils sont de petite dimension, ne dépassant pas les $35 \mathrm{~mm}$ de haut. Quatre ont été réalisés à partir d'un éclat épais (entre 5 et $36 \mathrm{~mm}$ d'épaisseur). C'est la face d'éclatement qui a servi de plan de frappe. Sur les six nucléus entiers, cinq sont de forme pyramidale avec une seule table de débitage. Le nombre de négatifs dépasse rarement six, avec en code opératoire 1-2-1-2-etc., ou 1-2-3-2-1, ou encore 1-2-3-4. Ce qui signifie que les enlèvements partent soit des flancs vers l'intérieur du nucléus soit d'un flanc vers l'autre. Il s'agit donc d'un débitage semi-tournant. Les négatifs des derniers enlèvements sont allongés (lames/lamelles?). Le septième est multidirectionnel avec quatre plans de frappe et trois tables de débitage, les derniers enlèvements sont des éclats. Les nucléus brûlés dominent le corpus.

Il y a 55 supports lamino-lamellaires (15 lames, 40 lamelles), fragmentés dans $75 \%$ des cas. Néanmoins, l'observation des talons présents donne un aperçu de la technique de percussion. Ainsi, les talons filiformes sont-ils souvent infra-millimétriques avec une forte abrasion de la corniche voire un doucissage. Les angles entre le bord du plan de frappe et la table de débitage oscillent entre $70^{\circ}$ et $80^{\circ}$. Il semble que la percussion directe tendre soit dominante. Les pièces entières ne dépassent pas les $30 \mathrm{~mm}$ de long. L'indice d'allongement est compris entre 2,5 et $3:$ nous avons donc des supports plutôt allongés adaptés à la réalisation de triangles ou de lamelles à bords abattus. Il semble par conséquent que les supports d'016 entrent dans les modules recherchés pour la fabrication des armatures. Les nervures des négatifs des précédents enlèvements sont sinueuses. Les négatifs vont par deux ou trois, voire quatre exceptionnellement.

Éclats, esquilles, fragments. 016 est le secteur qui comprend le plus de fragments : 1447 cassons, dont 125 esquilles, et 29 cupules thermiques, pour 322 éclats entiers. Près de $80 \%$ d'entre eux ont été chauffés. $46,3 \%$ sont inférieurs à $5 \mathrm{~mm}$ et $43,5 \%$ ont une dimension maximale comprise entre 5 et $10 \mathrm{~mm}$. Il est probable que la faible dimension des fragments et leur nombre résultent de l'action de la chauffe dont ils portent les stigmates. Outre le silex, d'autres roches sont présentes : grès, et aussi calcaire, cristal de roche, ophite, quartzite, sous forme de petits éléments. Sur les 322 éclats de silex entiers mesurés, plus de $60 \%$ sont brûlés/chauffés, les autres étant non altérés. Les éclats de 5 à $10 \mathrm{~mm}$ sont majoritaires $(40,4 \%)$ suivis par ceux entre 10 et $20 \mathrm{~mm}(23,7 \%)$ et ceux inférieurs à $5 \mathrm{~mm}(20,8 \%)$. Les cupules thermiques sont largement inférieures à $10 \mathrm{~mm}$. Ajoutons également qu'il n'y a aucune pièce corticale. Il s'agit donc de produits de plein débitage. La taille des produits, le fort taux de fragmentation et leur état de surface, ainsi que la présence de cupules thermiques, incitent à considérer 016 comme une fosse de rejet. 


\section{5 - Synthèse des données lithiques}

L'analyse technologique de l'industrie met en évidence une fréquentation au Premier Mésolithique, dont les témoins ont été reconnus dans les trois secteurs fouillés. Le Second Mésolithique/Néolithique ancien ne se manifeste que de façon discrète, dans le vestibule remanié. Les témoins des activités cynégétiques dominent.

\subsection{1 - Le Premier Mésolithique est dominant sur l'ensemble du site}

Les triangles isocèles et les scalènes, qui sont plutôt élancés et dont la moitié porte une retouche du troisième côté, rattachent les trois secteurs étudiés au Premier Mésolithique. Ils mobilisent des supports de petit module dont les bords et les nervures ne sont ni réguliers ni parallèles. La présence de triangles à caractères évolués évoque le Mésolithique ancien avancé (Mésolithique ancien, voire moyen) même si tous les critères habituels à cette période (l'hypermicrolithisme notamment) ne sont pas ici réunis. Ainsi, les triangles entiers ont-ils une longueur supérieure à $10 \mathrm{~mm}$ (entre 10 et $18 \mathrm{~mm}$ ) à l'exception de trois triangles entre 8 et $10 \mathrm{~mm}$ de long, et n'ont donc pas un caractère pygmée.

Pointes sur troncature, pointes étroites à bords abattus, et lamelles étroites à bords abattus confortent cette détermination.

Certes les microburins sont peu nombreux, mais bien présents. Ce procédé d'obtention des armatures n'était d'ailleurs pas d'emploi exclusif, et d'autres éléments témoignent aussi du façonnage des armatures (flexion des lamelles à coches). L'observation des supports lamino-lamellaires indique une percussion directe plutôt tendre sur des pièces étroites et peu épaisses. Ces types de supports s'inscrivent, comme les nucléus, dans les standards techniques du Premier Mésolithique.

\subsection{2 - Le Second Mésolithique est très discret}

47 Ses indices sont faibles et présents uniquement dans le vestibule: un trapèze asymétrique à retouches directes et une flèche de Montclus qui signalent une étape tout à fait finale du Mésolithique ou le Néolithique ancien. Les caractères marquants de cette phase ne se retrouvent sur aucune autre pièce retouchée: pas de retouches de style Montbani, pas de support large régulier, pas de percussion indirecte. Pas non plus d'armatures géométriques « évoluées " à retouche rasante et inverse, ni de pointes à base transverse. Cette carence contraste avec la bonne représentation de cette phase au plan régional (fig. 1), tant dans la plaine landaise au nord de l'Adour (Gaillèbes-2, Merlet et Miquéou 2009), que dans les Pyrénées versant nord (Poëymau) et sud (Aizpea, Zatoya).

\subsection{3 - Des traces d'un Néolithique ancien}

Enfin l'identification, dans le vestibule, de trois segments du Bétey marque une nouvelle fréquentation de la cavité au Néolithique ancien. En effet, ces armatures en double-biseau apparaissent avec le Néolithique ancien dans un espace géographique centré sur l'Aquitaine et le nord-ouest de la Péninsule ibérique (Miquéou 2012). 


\section{2 - La grande faune et la mésofaune (JBM)}

\section{1 - Matériel et méthode}

49 Les trois ensembles archéologiques mésolithiques de Bourrouilla ont livré des vestiges osseux. Ici sont présentés en détail les vestiges issus de la fouille de J25 et J26 ainsi que le matériel découvert dans la fosse de 016. Le lot des pièces du vestibule a fait l'objet d'une observation rapide qui a démontré la diversité de la faune. Elle comprend, non seulement des taxons semblables à ceux qui ont été identifiés dans les secteurs biens conservés, mais aussi d'autres taxons tel que des Bovidés et du Chamois, ainsi que des restes de chouette Harfang dont la «signature » magdalénienne était plus probable. De fait, ces restes n'ont pas été davantage étudiés.

La totalité de la faune a été analysée en utilisant la collection de comparaison du laboratoire PACEA (Université de Bordeaux). Les restes ont été observés à l'aide d'une loupe monoculaire (grossissement $\mathrm{x}$ 10) afin d'enregistrer le maximum de traces anthropogéniques ou d'agents taphonomiques post-dépositionnels. Le tout a été consigné dans une base de données accessible sur simple demande.

\section{2 - Résultats}

51 Au total, dans les deux locus étudiés, 217 restes ont été exhumés et coordonnés (tabl. 3). Ils proviennent pour les deux tiers des cas (NISP = 144) de la fosse du carré 016, le dernier tiers ayant été trouvé dans les carrés J25 et J26. La majorité des restes coordonnés n'a pu être identifiée au niveau spécifique en raison d'une intense fragmentation couplée à un mauvais état de conservation général.

Les vestiges identifiés sont essentiellement des restes d'ongulés occupant des milieux boisés. Parmi les restes déterminés spécifiquement, on retrouve le Cerf élaphe (36\%), le Chevreuil (25,6 \%) et le Sanglier (23,2\%). Un reste de Cheval est signalé (J25 n 253) : sa présence est inattendue sans être totalement inhabituelle, puisque des vestiges de ce type sont connus dans le Bassin parisien à la même époque (Ch. Leduc, com. pers. à MD). La mésofaune se compose de restes de Blaireau, de Martre, de Hérisson et de Castor.

Malgré la faible taille de l'échantillon analysé, il a été possible d'identifier différentes portions squelettiques allant de la tête jusqu'au bas des pattes et ce, pour les secteurs de fouille étudiés (tabl. 4). Ainsi, rejoignons-nous les premiers résultats obtenus par S. Costamagno (in Dachary et al. 2003) ; l'ensemble osseux de la fosse ne correspond pas au dépôt de quartiers de viande mais semble plutôt correspondre au dépôt de déchets de boucherie.

Exception faite du Hérisson, du Castor et des restes de microfaune, l'ensemble des restes analysés porte des traces anthropogéniques témoignant d'un apport par l'Homme. Ces traces se déclinent en traces de découpe (33/217) attestant le prélèvement de la viande, traces de raclage $(3 / 217)$ témoignant du retrait des derniers lambeaux de chair ou des tendons, de cône de percussion (8/217) pour extraire la moelle des os longs, et traces de brûlure (33/217). Ces dernières traces pouvant résulter de la cuisson de portions de carcasse ou d'une élimination des déchets dans un foyer. Ici, le faible nombre de restes ne nous permet pas de retenir une hypothèse plutôt qu'une autre. 
Parmi les restes portant des traces de découpe, il est intéressant de s'attarder sur l'un des vestiges de Blaireau. Il s'agit d'un fragment de coxal droit de blaireautin (fig. 7a). Par comparaison avec les squelettes modernes dont l'âge au décès est connu, nous retiendrons que l'individu de Bourrouilla a été tué autour des mois de mai-juin soit en milieu de bonne saison. Cette observation semble être corroborée par l'estimation de l'âge que nous avons pu faire sur les restes des trois principaux ongulés (tabl. 3).

Tableau 3 - Spectre faunique identifié et nombre minimum d'individus calculé pour les principaux taxons.

\begin{tabular}{|l|c|c|}
\hline Taxons & NISP & NMI \\
\hline Cheval & 1 & 1 adulte \\
\hline Cerf & 31 & 1 adulte, 1 jeune adulte \\
\hline Chevreuil & 22 & 1 adulte, 1 jeune adulte \\
\hline Sanglier & 20 & 1 adulte, 1 jeune adulte \\
\hline Blaireau & 8 & 1 adulte, 1 jeune adulte \\
\hline Martre & 2 & 1 adulte \\
\hline Hérisson & 1 & 1 jeune adulte \\
\hline Castor & 1 & 1 adulte \\
\hline Microfaune & 3 & - \\
\hline Ongulé 1 & 3 & - \\
\hline Ongulé 1/2 & 7 & - \\
\hline Ongulé 2 & 19 & - \\
\hline Ongulé 2/3 & 5 & - \\
\hline Ongulé 3/4 & 1 & - \\
\hline Mammifère indét. & 93 & 12 individus \\
\hline Total & 217 & - \\
\hline
\end{tabular}

56 Concernant les traces de découpe observées, tant sur la Martre que sur le Blaireau, par comparaison avec un référentiel de boucherie (Mallye 2007, 2011), l'hypothèse d'une exploitation de ces animaux pour leur fourrure peut être rejetée; ces traces correspondent à des actions de décarnisation (fig. $7 b$ ). Dès lors, la viande ayant été consommée, il n'est pas possible de statuer sur la conservation ou non de la fourrure. 


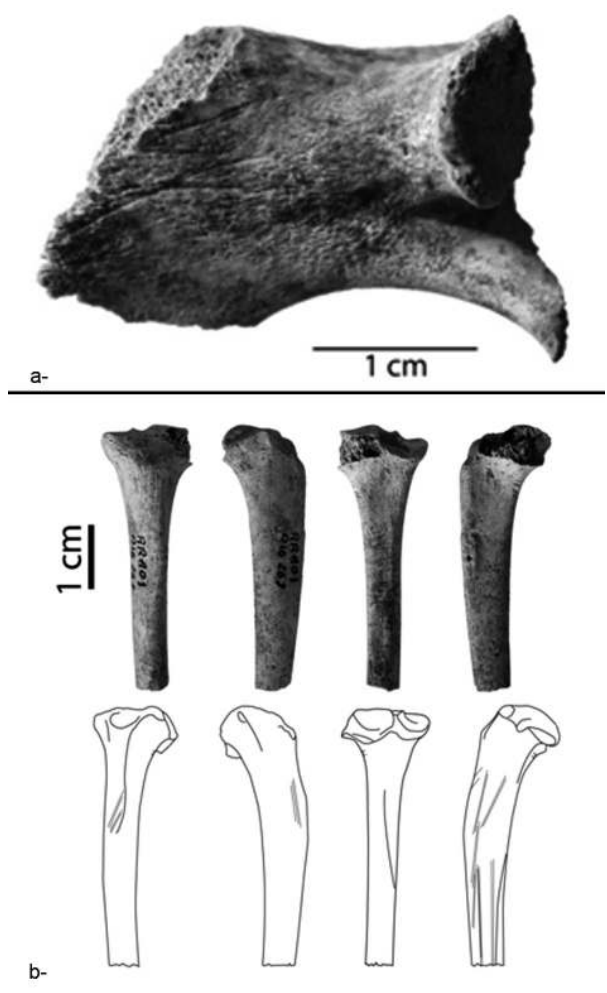

a- Fragment de coxal droit de blaireautin portant des traces de décharnement. b- Tibia gauche de Martre portant plusieurs traces anthropogéniques. Le relevé de ces traces (bas) indique des gestes de décarnisation (@ J.-B. Mallye).

Pour conclure, bien que le matériel exhumé soit en faible nombre, il nous permet de montrer l'origine anthropique des restes de faune mésolithique de Bourrouilla. Le décompte des pièces indique la présence d'au moins 12 individus, se rapportant majoritairement à des espèces évoluant dans des environnements forestiers. Il a pu être démontré que les restes exhumés de la fosse correspondent au dépôt de déchets de boucherie. Enfin, sur la base de l'âge au décès des individus identifiés, une occupation du gisement en milieu de bonne saison est hautement probable.

\section{3 - L'ichtyofaune de la fosse de 016 (OLG)}

\section{1 - Le site dans son contexte hydrogéographique actuel}

Le gisement d'Arancou domine la rive droite du Lauhirasse, ce ruisseau est un affluent de la rive droite de la Bidouze. Dans la zone qui nous intéresse, cette rivière est aujourd'hui caractérisée par un lit d'environ 25 mètres de large assez peu profond. Les poissons que l'on y trouve sont essentiellement des poissons blancs (Chevesne, Vandoise, Barbeau, Gardon). L'Anguille y est bien sûr présente. Le fait que le Muge (migrateur amphibiotique agamadrome) y remonte est à souligner. Il démontre que la Bidouze est un axe de migration commode. De nos jours le Saumon atlantique ne l'utilise toutefois pas. La Bidouze rejoint la rive gauche de l'Adour face à l'île de Mirepech, à peu de distance en aval de la confluence Adour-Gaves Réunis. 
De plus, le site d'Arancou n'est pas très éloigné du Gave d'Oloron. Le chemin le plus court pour y parvenir mène à la rive gauche du Gave, aux environs d'Escos, à quelques kilomètres en amont des sites magdaléniens de la falaise du Pastou à Sorde-l'Abbaye.

Tableau 4 - Éléments squelettiques identifiés chez le Cerf, le Chevreuil et le Sanglier d'une part dans le secteur d'entrée de la grotte (J25 et J26) et d'autre part dans la fosse (carré 016).

\begin{tabular}{|l|c|c|c|c|c|c|c|}
\hline & \multicolumn{3}{|c|}{ Secteur entrée } & \multicolumn{3}{c|}{ Fosse O16 } & \multirow{2}{*}{ Total } \\
\hline Anatomie & Cerf & Chevreuil & Sanglier & Cerf & Chevreuil & Sanglier & \\
\hline Bois & 1 & - & - & - & - & - & 1 \\
\hline Fragments crâniens & - & - & - & 1 & - & 3 & 4 \\
\hline Mandibule & - & - & - & 1 & 2 & - & 3 \\
\hline Dents isolées & 2 & - & 6 & 1 & 1 & 1 & 11 \\
\hline Vertèbres & - & - & - & 2 & 4 & 2 & 8 \\
\hline Côtes & - & - & - & 3 & - & - & 3 \\
\hline Scapula & 1 & - & - & 1 & - & - & 2 \\
\hline Humérus & - & - & 1 & 2 & 1 & - & 4 \\
\hline Radius & 1 & - & - & - & - & - & 1 \\
\hline Radio-ulna & - & - & - & 1 & - & - & 1 \\
\hline Carpiens & 1 & 1 & - & - & - & 2 & 4 \\
\hline Métacarpiens & - & - & - & - & 2 & 2 & 4 \\
\hline Coxal & 1 & - & - & - & 1 & 1 & 3 \\
\hline Fémurs & - & - & - & 2 & 3 & - & 5 \\
\hline Tibia & 1 & 1 & - & 2 & 2 & - & 6 \\
\hline Tarsiens & 1 & - & - & - & - & 1 & 2 \\
\hline Métatarsiens & 1 & - & - & - & 2 & - & 3 \\
\hline Phalange 1 & - & - & - & 1 & 1 & - & 2 \\
\hline Phalange 2 & - & - & - & - & - & 1 & 1 \\
\hline Phalange 3 & - & - & - & - & 1 & - & 1 \\
\hline Phalange & - & - & - & 1 & - & - & 1 \\
\hline Sésamoïde & - & - & - & 2 & - & - & 2 \\
\hline Métapode & 1 & - & - & - & - & - & 1 \\
\hline \multicolumn{1}{|c|}{ Total } & - & - & - & 20 & 20 & 13 & 73 \\
\hline & - & - & - & & & 2 \\
\hline
\end{tabular}

\section{2 - Description des restes d'ichtyofaune}

La fosse recelait 134 vestiges de poissons dont 114 (80 \%) sont déterminables (fig. 8). Ils se répartissent entre Cyprinidés (97 restes, soit $86 \%$ des déterminés), Salmonidés (sept restes, soit $6 \%$ des déterminés), Esocidés (quatre restes, soit $4 \%$ des déterminés) et Anguillidés (quatre restes, soit $4 \%$ des déterminés). La très grande majorité des pièces était composée de vertèbres auxquelles s'ajoutent quelques arcs pharyngiens de Cyprinidés complets ou fragmentaires. Les morceaux d'os crâniens et les tronçons de côtes sont restés indéterminés. 
Figure 8 - L'ichtyofaune de la fosse du carré 016.

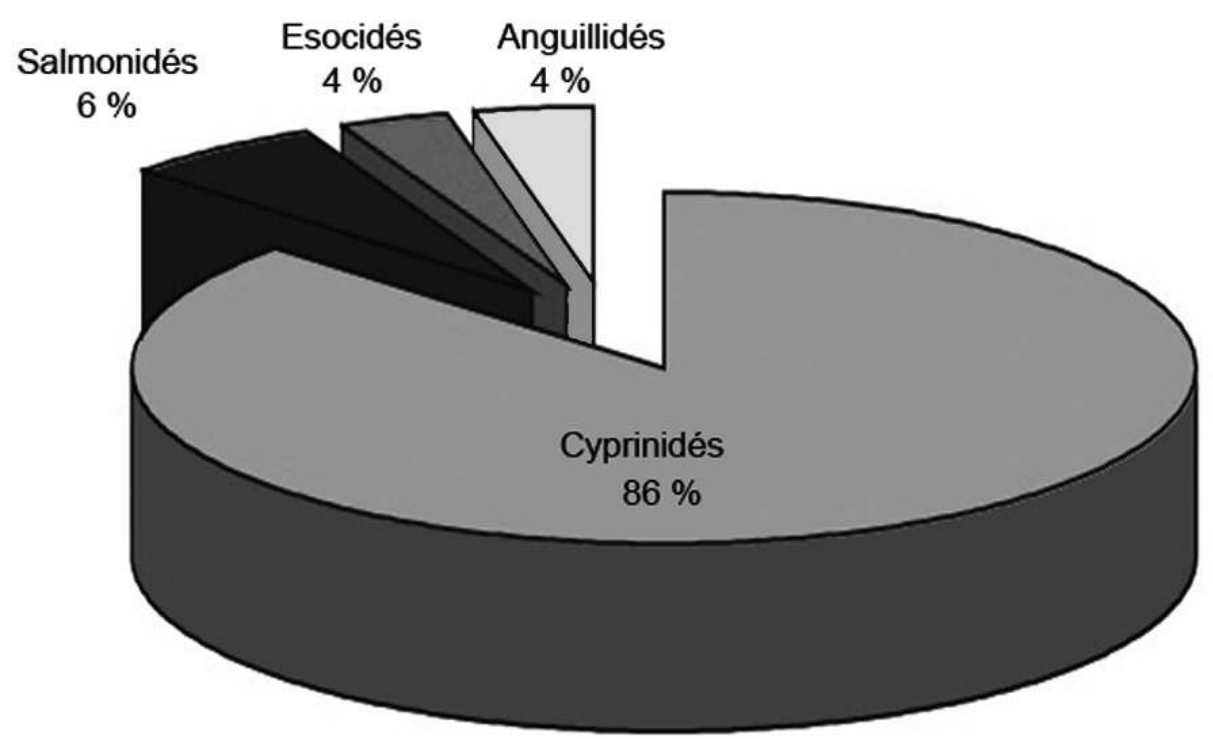

\subsection{1 - Les Cyprinidés}

\section{Les Leuciscus sp. (Chevesne, Vandoise)}

Les restes du genre Leuciscus se taillent la part du lion avec 93 os. S'ils se rapportent au Chevesne (Leuciscus cephalus) pour leur grande majorité, la présence de quelques vertèbres de Vandoise (Leuciscus leuciscus) n'est toutefois pas à écarter. Les poissons de ce genre sont un classique des ichtyofaunes pléistocènes et holocènes. Très ubiquistes, ils font partie des résidents des zones à Truite, à Ombre, à Barbeau et à Brême de Huet. En conséquence, ils n'ont guère de signification en ce qui concerne les milieux. Ils préfèrent les eaux courantes aux eaux stagnantes. La reproduction aux mois de mai-juin réunit de nombreux individus et donne lieu à des ébats bruyants (Spillmann 1961 - p. 136).

Soixante-douze vertèbres m'ont permis de faire des évaluations quant à la longueur des sujets. La majorité des Leuciscus sp. a été prise alors qu'ils atteignaient des tailles variant entre $15 \mathrm{~cm}$ et $25 \mathrm{~cm}$, le plus grand nombre s'approchant d'une longueur voisine de $20 \mathrm{~cm}$.

Trente-neuf vertèbres ont autorisé la lecture de la saison de capture par comparaison avec une série de référence (Le Gall 1999, 2003). Pour l'utilisation en archéologie, et compte tenu des incertitudes pesant sur la durée de chaque saison, il est difficile de préciser au-delà de bonne ou mauvaise saison (assez facilement séquencée en début, pleine et fin). Les indications en termes de mois sont donc à considérer à titre indicatif, facilitant le report des observations dans nos échelles actuelles de mesure du temps.

La quasi totalité des Leuciscus a été capturée au cours de la bonne saison (avrilnovembre). La mauvaise saison (novembre-mars) n'est représentée que par un élément, provenant peut-être d'un individu pathologique. Le début de la bonne saison (avriljuin) et la pleine bonne saison (juin-septembre) sont respectivement représentés par 24 et huit éléments.

Les Barbus sp. (Barbeau fluviatile et Barbeau méridional) 

massives dotées de fortes pré et post-zygapophyses supérieures (Le Gall 1982, 1984, 1999). Malheureusement, ces éléments résistent assez mal à l'enfouissement. Quoi qu'il en soit, six vertèbres évoquent fortement la présence de ce genre.

$\mathrm{Vu}$ la position géographique du site (versant nord-ouest de la chaîne pyrénéenne), il s'agit vraisemblablement du Barbeau fluviatile (Barbus barbus). Sa présence à l'Holocène n'est pas vraiment étonnante : il a déjà été signalé dans la couche 5 de la grotte de Pégourié (Lot) dans un contexte "Azilien évolué » (Le Gall 1995, 1999). Sa présence est toutefois intéressante car elle marque le retour des ichtyofaunes « chaudes » sur notre territoire (Le Gall 2008, 2010) et la mise en place de nos actuelles populations de poissons d'eaux douces. Le Barbeau fluviatile se rencontre dans les zones à Ombre, à Barbeau et à Brême de Huet. Cependant, ses préférences vont à des eaux pures, relativement vives, à fonds caillouteux ou sableux. Au moment du frai, fin avril-mi juin, les barbeaux effectuent des migrations, parfois d'une certaine ampleur, afin de trouver les substrats leur convenant.

Sur les six vertèbres attribuables à ce genre, quatre seulement ont permis de faire une évaluation de taille des individus. Par rapport à l'espèce, vraisemblablement le Barbeau fluviatile (Barbus barbus), ces poissons sont de petites tailles (tailles courantes entre 20 et $60 \mathrm{~cm}$, mais ils peuvent atteindre 80 à $100 \mathrm{~cm}$ ). Dans le Mésolithique de Bourrouilla, la longueur la plus courante est $20 \mathrm{~cm}$. Ce tri dans les dimensions paraît être le même que chez les Leuciscus qui peuvent aussi atteindre des tailles importantes (facilement 50 à $60 \mathrm{~cm}$ ).

Seules trois vertèbres ont autorisé la lecture de la saison de capture. Les indications de capture en début de bonne saison peuvent, comme chez Leuciscus, correspondre à la période du frai, ou au déplacement vers l'amont qu'effectuent les barbeaux à cette occasion.

Une question subsiste cependant : pourquoi aussi peu de prises alors que les barbeaux se déplacent en bancs lors de leur migration vers les frayères?

\subsection{2 - Les Salmonidés ; le genre Salmo}

Très peu nombreux dans cette fosse mésolithique, leurs os sont au nombre de sept, tous attribuables à la Truite de rivière (Salmo trutta fario). C'est un poisson résident qui apprécie les eaux vives et bien oxygénées. On la trouve dans les zones à Truite, à Ombre et à Barbeau de Huet. Avant le frai, novembre-décembre, les reproducteurs se livrent à des migrations afin d'accéder aux substrats idéaux, souvent en tête de bassin. Seules quatre vertèbres ont permis une estimation de la longueur des poissons et deux seulement celle de la saison de capture : deux pièces se rapportent à des poissons de 20 à $25 \mathrm{~cm}$ dont un pris en début de bonne saison, une autre à une truite de 30 à $35 \mathrm{~cm}$. La plus grande faisait 45 à $50 \mathrm{~cm}$, ce qui indique la présence d'un grand reproducteur. Il est par ailleurs intéressant de noter que celui-ci a été pris en fin de bonne saison, période où ces poissons se livrent à des migrations vers l'amont en direction de leurs frayères. 


\subsection{3 - Les Esocidés}

71 Ils sont représentés dans nos contrées par un seul genre et une seule espèce : le Brochet (Esox lucius). Ce carnassier est un poisson résident de nos eaux douces. Il effectue des migrations avant le frai et se reproduit, en fonction des régions, à l'extrême fin de la mauvaise saison (mars) ou tôt dans la belle saison (avril). La température optimale pour la ponte se situe aux alentours de 7 à 10 degrés. Le Brochet se rencontre dans de nombreux endroits; des milieux faiblement salés de la Baltique et de la «zone à flet ». Cependant, c'est surtout un poisson classique de la zone à Brême de Huet. Il ne craint toutefois pas les eaux froides et son seul handicap est de ne pas supporter des courants trop violents. On le trouve cependant fréquemment dans la « zone à Barbeau », parfois dans la «zone à Ombre ». Quelques individus arrivent même à subsister dans des recoins calmes de la "zone à Truite " mais c'est un phénomène récent consécutif à la création d'étangs de loisirs à partir de petits cours d'eau de la « zone à truite».

Sur les quatre vertèbres attribuables à ce genre, trois ont fourni des indications de dimensions : une aux alentours de $40 \mathrm{~cm}$ et deux correspondant à un ou des poisson(s) de 50 à $55 \mathrm{~cm}$. Dans cette dernière catégorie, un vestige indique le début de la bonne saison. S'agit-il d'un reproducteur en train de redescendre vers l'aval après le frai?

\subsection{4 - Les Anguillidés}

Le seul représentant dans nos eaux douces, l'Anguille (Anguilla anguilla), est représenté par quatre vertèbres retrouvées dans la fosse.

L'Anguille, migrateur amphihalin génésique catadrome, naît dans la Mer des Sargasses. Sous formes larvaires (leptocéphales), les anguilles se laissent porter jusqu'à nos côtes. Leur voyage transatlantique est assuré par le Gulf Stream et la dérive Nord-Atlantique qui, se répartissant en plusieurs branches, déterminent trois populations: une septentrionale pour l'Islande et la Norvège, une centrale pour les Iles Britanniques et les côtes françaises atlantiques, et une méridionale pour le Portugal, le Maroc et le détroit de Gibraltar. Les leptocéphales se transforment en civelles à l'entrée des fleuves, au tout début de leur migration anadrome (montée). Au cours de celle-ci, les civelles se transforment en Anguillettes puis en Anguilles jaunes. Les remontées en eaux douces (octobre à avril), ainsi que les multiples transformations simultanées que subissent les civelles, sont conditionnées par de très nombreux facteurs. Le facteur clé est tout de même la température : si elles recherchent des eaux fraîches, les civelles respectent des « seuils », 7-9 ${ }^{\circ} \mathrm{C}$ en Europe du Nord, 11-22 ${ }^{\circ} \mathrm{C}$ pour l'Arno (Bruslé et Quignard 2001).

Il semble que les anguilles déterminent leur sexe en eaux douces et que les mâles se caractérisent par une taille inférieure aux femelles. De plus, la répartition des sexes le long des cours d'eau montre que les mâles restent fréquemment à proximité des zones estuariennes alors que les femelles, au plus fort rhéotropisme, remontent très en amont jusqu'à la "zone à truite ». La durée de leur séjour en eaux douces est variable : 8 à 15 ans pour les mâles et 10 à 18 ans pour les femelles. Au printemps, elles se préparent à l'avalaison pour rejoindre l'aval des cours d'eau. C'est le moment où les individus réfugiés en eaux closes cherchent à rejoindre les eaux vives. Pour cela, elles sont capables de se déplacer à terre, dans des prairies humides. A la fin de l'été, les anguilles sont dans les parties avales des cours d'eau et subissent des transformations physiologiques. C'est la transformation des anguilles jaunes en anguilles argentées. Les anguilles quittent les fleuves pour s'engager en mer à la fin de l'automne et au début de 
l'hiver. Elles mesurent alors de 30 à $50 \mathrm{~cm}$ pour les mâles et de 40 à $100 \mathrm{~cm}$ pour les femelles.

Durant la seconde partie du dernier Glaciaire, le Tardiglaciaire et l'Holocène, la remontée de l'Anguille vers le nord de l'Europe témoigne vraisemblablement d'un réchauffement progressif agissant sur les eaux océaniques (Kettle et al. 2008) complétant la mise en place du Gulf Stream et, successivement, des trois branches de la dérive Nord-Atlantique (méridionale, centrale puis septentrionale).

Sur les quatre vertèbres d'anguilles découvertes en 016, trois ont permis de mettre en évidence la présence d'un individu de 25 à $30 \mathrm{~cm}$ capturé en bonne saison et de deux autres, de respectivement 60 à $65 \mathrm{~cm}$ et de 90 à $95 \mathrm{~cm}$. Ce dernier a été capturé en début de bonne saison.

\section{3 - Quel agent accumulateur ? Quel mode de capture?}

L'observation de "picking» (traces de dissolutions arrondies, souvent attribuées à l'action des sucs digestifs animaux) sur les faces articulaires de certaines vertèbres nous a poussé à nous interroger sur l'origine des restes de poissons découverts.

Des mammifères peuvent être à l'origine de certaines accumulations de restes de poissons. C'est notamment le cas de la Loutre, mais le site est trop éloigné des cours d'eau pour avoir été occupé par cet animal.

Figure 9 - Taille des poissons de la fosse mésolithique (016).

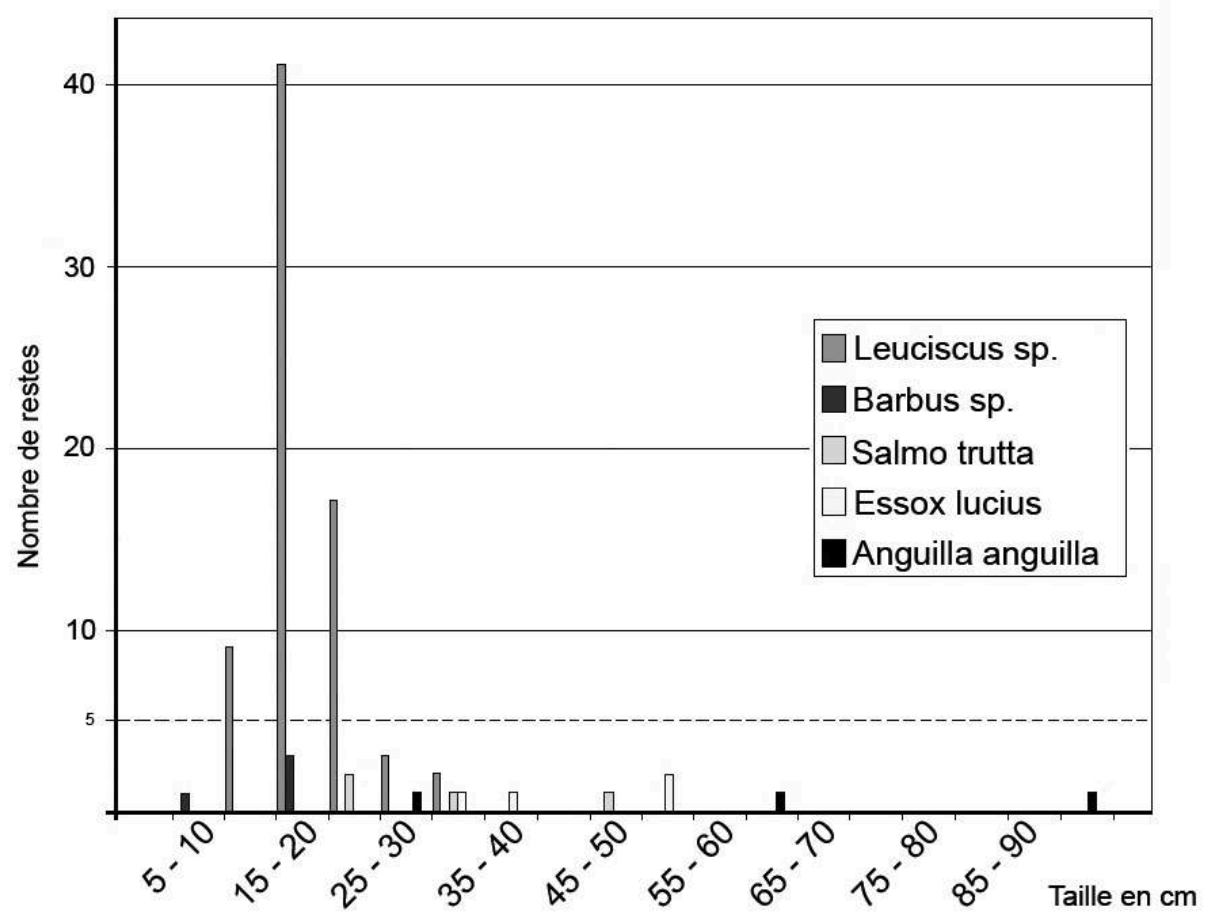

Le Choucas des tours (Corvus monedula) peut être responsable d'accumulation de restes de poissons (Le Gall 2011), mais il niche dans des falaises et c'est à l'aplomb de celles-ci qu'il rejette ses déchets. La localisation de la fosse du carré 016, à une dizaine de mètres du porche d'entrée, est incompatible avec cette hypothèse. De même, si 
l'ichtyofaune mésolithique découverte à Arancou est très voisine de celle consommée par le Grand-Duc, avec une prédominance des Cyprinidés, les dimensions des poissons mésolithiques de Bourrouilla ne s'accordent pas avec celles des proies de ce rapace nocturne.

Par ailleurs de nombreux arguments vont dans le sens d'une accumulation d'origine anthropique :

- l'ensemble du contenu de la fosse est fortement anthropisé. L'outillage ainsi que les produits de débitage sont nombreux;

- la mégafaune associée est riche, toujours en tenant compte de l'exiguïté de la fosse, en espèces et en nombre de restes. Elle est aussi caractéristique du spectre faunique exploité par les Mésolithiques (Cerf, Chevreuil, Sanglier...) et ces vestiges correspondent à des restes de boucherie ;

- L'observation des stades d'éruptions dentaires indique des saisons de chasse de bonne saison qui sont globalement conformes aux résultats obtenus à partir de l'ichtyofaune.

81 La taille des poissons correspond à la limite «basse » de ceux retrouvés en contexte azilien (Pont d'Ambon par exemple) où l'emploi d'engins de type nasses avait été évoqué.

Rappelons le mode de fonctionnement de ces pièges : une nasse est un objet fabriqué à partir d'éléments végétaux souples assemblés par vannerie. Elle est constituée d'un panier, destiné à retenir captifs les poissons qui ont pénétré à l'intérieur du piège, et d'un goulet d'entrée, généralement en forme de cône. Ce goulet est conçu de manière à autoriser le passage des poissons en direction de l'intérieur du panier et à empêcher leur sortie. Les prises seront donc « calibrées » en fonction de la plus petite ouverture du goulet. L'élément déterminant de cette sélection sera donc l'épaisseur des poissons. Ce dernier critère est variable en fonction des saisons, notamment, et à mettre en rapport direct avec la richesse en nourriture du milieu aquatique. Même si le critère " épaisseur » est variable, il reste tout de même assez étroitement lié au caractère «longueur » lequel nous demeure accessible. Bref une nasse "pêchera » en effectuant un tri dans la taille des proies.

83 C'est exactement ce que l'on observe dans le Mésolithique de «Bourrouilla » (fig. 9). Ce graphique nous montre que la majorité des poissons sont des Cyprinidés des genres Leuciscus et Barbus. Pour ces poissons, la grande majorité des individus affiche des longueurs de 15 à 25-30 cm, c'est-à-dire des tailles moyennes. Les gros individus (40-50 $\mathrm{cm})$ n'ont pas été capturés alors qu'ils étaient forcément présents dans le cours d'eau. En ce qui concerne les anguilles, poissons longilignes s'il en fut, elles sont tout à fait capables de pénétrer dans des endroits forts exigus. Tous les arguments vont dans le sens de l'utilisation de tels pièges par les Mésolithiques de la grotte de Bourrouilla.

Or, si l'existence de filets (utilisation inconnue pour le moment) semble remonter au Gravettien (Adiovaso et al. 1999; Sofer et al. 2000), l'apparition des nasses est sans doute un peu plus ancienne que l'Azilien. Une preuve en est fournie par un ensemble de gravures exécutées au Magdalénien supérieur. Le galet de quartzite qui leur sert de support à été découvert sur le site de plein air de La Honteyre (Le Tuzan, Hautes Landes girondines). Une des gravures (Le Gall et al. 2006) du galet figure un probable Cyprinidé. La partie antérieure du corps de ce dernier paraît contenue dans un ensemble de tracés réalisés postérieurement. Simple vers la tête du poisson, celui-ci se complique singulièrement vers la partie médiane de ce dernier, constituant alors un véritable réseau en résille. Exprimé par des losanges sur les flancs du poisson, il est 
constitué de traits courbes entrelacés au niveau de son dos. De tels entrelacs pourraient, de manière assez schématique mais néanmoins parlante, évoquer un objet réalisé en vannerie (nasse, panier à pêche, ...).

Si la capture à l'aide de nasse semble vraisemblable dans le cas du Mésolithique de Bourrouilla, il reste à expliquer la prédominance des Cyprinidés dans le spectre des prises. Cette spécialisation correspond probablement à une option saisonnière. En effet, le Chevesne (Leuciscus cephalus cephalus) atteint et dépasse les $60 \mathrm{~cm}$ et fraye en maijuin à l'occasion de rassemblements bruyants de nombreux individus. La Vandoise (Leuciscus leuciscus) mesure de 15 à $25 \mathrm{~cm}$ et fraye de nuit en février-mai après une légère migration.

Enfin, le Barbeau fluviatile (Barbus barbus) est un poisson de 20 à $60 \mathrm{~cm}$, (jusqu'à $1 \mathrm{~m}$ parfois) qui fraye d'avril à mi-juin après des migrations de quelques kilomètres. Si nous examinons le graphique regroupant les saisons de capture de l'ichtyofaune de la fosse O16 (fig. 10), il apparaît que la majorité des prises ont été réalisées en début de bonne saison sur des Leuciscus et des Barbus de 20 à $25 \mathrm{~cm}$. Il s'agit donc de barbeaux en migration et de vandoises en pleine reproduction. Pour ce faire, la meilleure technique est la nasse pêchant de nuit, c'est ce que semble confirmer également la capture d'anguilles.

87 Au total, il semble que l'ichtyofaune de la fosse mésolithique de la grotte de Bourrouilla a bien une origine anthropique. L'usage de nasses est hautement vraisemblable si l'on considère la régularité des longueurs des poissons capturés pour l'essentiel en début de bonne saison. En outre, la diversité des états de surfaces du matériel plaide pour une accumulation en plusieurs étapes.

Figure 10 - Saisons de captures de l'ichtyofaune mésolithique de 016.

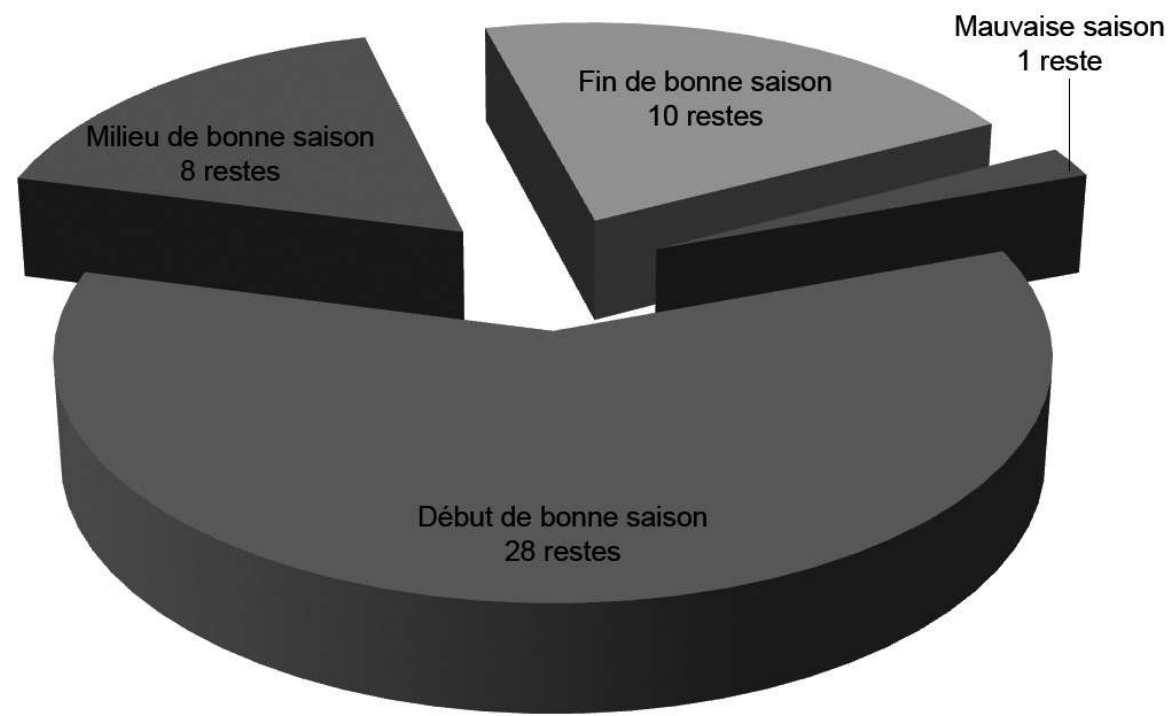




\section{4 - L'avifaune de la fosse de 016 (A. E.)}

88 La fouille de la fosse mésolithique du carré 016 a livré 13 restes d'oiseaux dont 12 sont déterminables. La Buse variable (Buteo buteo) est le taxon dominant puisque 11 pièces lui sont attribuables. Ces vestiges correspondent à au moins deux individus dont une femelle exceptionnellement grande.

89 L'étude archéozoologique de ces restes montre qu'un seul porte des traces anthropiques. Il s'agit d'un fragment de radius où sont visibles des cut-marks. Ce vestige conduit à penser que la Buse fût chassée par les Mésolithiques. Pour autant, il n'est pas possible de savoir si cet animal est intervenu dans un cadre alimentaire, technique et/ou symbolique.

90 L'ultime reste déterminable est attribuable à la chouette Harfang (Nyctea scandiaca). Ce taxon est très bien représenté dans les niveaux magdaléniens de Bourrouilla, mais sa présence dans le piémont pyrénéen au cours de l'Holocène est plus qu'improbable. La fosse mésolithique ayant été creusée dans un ensemble sédimentaire contemporain des occupations magdaléniennes, il est vraisemblable que ce reste soit intrusif.

\section{5 - Datations radiocarbone}

91 Nous avons choisi de réaliser deux datations radiocarbone sur des charbons issus $d u$ carré 016. Confiés au laboratoire Beta Analytic, les deux échantillons ont subi le protocole standard Acide-Base-Acide. Les résultats sont résumés dans le tableau 5.

92 La lecture de ces résultats amène d'emblée deux constats: d'une part, ces deux datations sont recevables pour du Mésolithique, d'autre part, alors que les deux échantillons proviennent de la même US, interprétée comme une fosse de rejet, les datations sont différentes et les intervalles de confiance à 2 sigma ne se recoupent pas. C'est même l'échantillon le plus profond (016 317) qui fournit la date la plus jeune.

93 La comparaison avec d'autres datations du Mésolithique du sud-ouest de la France (tabl. 6 et fig. 11) oblige cependant à reconnaître que les industries à triangles constituant le Premier Mésolithique sont en général un peu plus anciennes que ce qu'indiquent les deux datations présentées ici. Toutefois, le sommet de la séquence Mésolithique de la Lède du Gurp à Grayan-et-L'Hôpital est daté de $7360 \pm 85$ et $7350 \pm$ 130 BP (Roussot-Larroque 2011) tandis que le Premier Mésolithique de la Grotte du Sanglier à Reilhac s'étend jusque vers 7500 BP (Séronie-Vivien et al. 2001). Notons aussi la date obtenue au Roc Allan (Sauveterre-La-Lémance) de 7625 \pm 80 BP (Detrain et al. 2006), très proche des résultats d'Arancou.

$94 \mathrm{Au}$ total, sans nier qu'un âge un peu plus ancien était vraisemblable compte tenu du matériel lithique mis au jour, les datations réalisées sont parfaitement acceptables, d'autant que l'on manque cruellement de point de comparaison pour les Pyrénées occidentales.

Tableau 5 - Datations radiocarbone de la fosse mésolithique 016.

\begin{tabular}{|l|l|l|l|}
\hline Désignation & Numéro de laboratoire & Age BP & Age Cal BC \\
\hline O 1687 & Beta-307295 & $7650 \pm 40$ & $6590 / 6580$ ou $6570 / 6440$ \\
\hline O 16317 & Beta-307296 & $7410 \pm 40$ & $6480 / 6220$ \\
\hline
\end{tabular}




\section{6 - Discussion}

\section{1 - Bilan des occupations} interprétations possibles :

- les charbons datés sont pollués par des infiltrations d'eau et donnent une datation erronée. Cette hypothèse est peu vraisemblable puisque d'autres datations obtenues sur les niveaux magdaléniens dans les carrés adjacents à 016 sont tout à fait conformes à ce que l'on pouvait attendre (Szmidt et al. 2009) ;

- l'association entre une industrie du Premier Mésolithique et les charbons datés témoigne d'une occupation plus récente du site et d'un réaménagement du sol d'habitat ayant amené au comblement de la fosse avec des matériaux d'âge varié. Les différences d'état de surface parmi l'ichtyofaune pourraient alors trouver une explication, de même que le fait que l'échantillon le plus profond (016 317) est celui qui fournit la date la plus jeune. L'absence de toute trace d'un Second Mésolithique dans la fosse est dans ce cas bien surprenante ;

- les datations correspondent effectivement à une ou plusieurs occupations datant du Premier Mésolithique. Les dates, très basses, indiqueraient alors un «retard» dans l'adoption des comportements techno-typologiques caractéristiques du Second Mésolithique, conformément au modèle récemment proposé par T. Perrin et al. (2009). Rappelons, en outre, que les dates du sud-ouest de la France qui peuvent être évoquées à titre de comparaison proviennent soit du nord de l'Aquitaine soit du Quercy.

Notons que les deux dernières hypothèses ne sont pas totalement incompatibles. On peut envisager un passage au Second Mésolithique plus tardif ici que dans d'autres régions, en même temps qu'un comblement de la fosse réunissant des vestiges d'occupations mésolithiques étalées dans le temps. 


\section{2 - Des données encore difficiles à situer dans leur contexte régional}

99 Les niveaux mésolithiques de la grotte Bourrouilla livrent des données non négligeables, d'autant que la connaissance du Mésolithique dans cette région souffre encore d'une sous-documentation. La précision de la fouille, qui a comporté un tamisage fin systématique, et la présence de vestiges osseux, qui font cruellement défaut sur les gisements de plein air, autorisent la perception de certaines des stratégies développées par les derniers chasseurs-collecteurs du piémont nordoccidental des Pyrénées.

100 Il n'existe pas de lien physique entre les trois locus, ce qui interdit de discuter d'une répartition spatiale des occupations. Cependant, la découverte d'une "structure en creux" à l'intérieur de la cavité permet de faire une comparaison avec des aménagements mis en évidence dans le nord de la France dans les vingt dernières années (cf. Ch. Verjux in Guesquière et Marchand 2010 - p. 112-113). L'étroitesse de la surface fouillée n'a pas permis d'apporter une documentation suffisante pour discuter du réemploi d'une structure antérieure ou de son rythme de comblement, tandis que l'absence de données sur des structures équivalentes dans un cadre géographique proche limite la discussion.

101 Cette série constitue en outre un jalon intéressant dans la distribution des sites mésolithiques de l'Aquitaine méridionale et occidentale, en raison de la situation géographique de la cavité, entre les zones montagneuses des Pyrénées au sud, les collines de Chalosse et la plaine landaise au nord.

Les Mésolithiques ont certainement développé des stratégies leur permettant d'exploiter alternativement, selon les saisons, les ressources de différents biotopes: ceux de la forêt, des rivières, de la montagne, voire du littoral océanique. Bourrouilla n'est qu'une étape très ponctuelle (mai-juin) sur un territoire parcouru en fonction des déplacements saisonniers. Le bon état de conservation de la faune permet de démontrer l'exploitation de deux milieux complémentaires: le milieu forestier, où l'avifaune est aussi chassée, et le milieu aquatique (poissons mais aussi moules d'eau douce, présentes dans la fosse 016).

103 Les données paléontologiques qui indiquent une chasse des ongulés de milieu forestier et, assez logiquement, l'industrie lithique, surtout composée d'armatures, témoignent de ces activités cynégétiques. De plus, on sait que ce sont les armatures qui nécessitent le plus de renouvellement et de réfection. Certes, la question est posée des outils servant à la découpe des carcasses et au raclage de la viande, ainsi qu'aux autres activités domestiques. Cependant, l'étude techno-typologique a mis en avant l'absence de certains éléments typologiques caractéristiques du Premier et du Second Mésolithique. Or, d'autres lacunes doivent être relevées, en dépit du nomadisme avéré des occupants de cette cavité :

- l'absence de matières lithiques autres que le silex du Flysch, collecté à peu de distance du gisement ;

- la rareté de l'outillage du fonds commun, comparativement à l'abondance en armatures. Ce sont les éclats et fragments qui dominent dans l'effectif des artefacts, ceux inférieurs à $5 \mathrm{~mm}$ sont d'ailleurs très nombreux. 
104 Comprendre la mobilité des groupes de chasseurs-cueilleurs au sein du piémont pyrénéen durant les premières phases du Mésolithique, nécessitera donc de fouiller d'autres sites pour avoir une vision à l'échelle d'un territoire de vie.

Tableau 6 - Inventaire des datations radiocarbone du Mésolithique du sud-ouest de la France (d'après Roussot-Larroque 2009).

\begin{tabular}{|c|c|c|c|c|}
\hline Site & Coucho & Róférence & Date BP & Date cal BC \\
\hline ARANCOU, grotte Bourrouilla & & Beta-307295 & $7650 \pm 40$ & 644016570 \\
\hline ARANCOU, grotte Bourrouilla & & Beta-307296 & $7410 \pm 40$ & $6220 ; 6390$ \\
\hline CIEUX, abri des Fóes & 84 & Ly-4534 & $9490 \pm 240$ & $8260 / 9020$ \\
\hline ClEUX, abri des Fées & 84 & Ly-4707 & $8850 \pm 270$ & $7730: 8100$ \\
\hline CIEUX, abri des Fóes & B4 sommot & Ly-4706 & $7535 \pm 110$ & $6300 ; 6450$ \\
\hline COSNAC, chez Jugie & 3b base & Ly-1652 & $8080 \pm 280$ & $6570 / 7450$ \\
\hline COSNAC, chez Jugie & $3 \mathrm{~b}$ base & Ly-1331 & $8040 \pm 260$ & 6590,7390 \\
\hline COSNAC, chez Jugie & 3b base & Ly-1651 & $7650 \pm 510$ & $6438 ; 6573$ \\
\hline 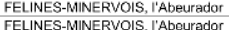 & & MC-2144 & $8740 \pm 90$ & $7590 / 7750$ \\
\hline FELINES-MINERVOIS, 'IAbourador & $x$ & Gif-6745 & $8740 \pm 90$ & 7430,7530 \\
\hline $\begin{array}{l}\text { GEAA, La Pierre Saint Louis } \\
\text { GRAANAN-t-L'H, L̇de do Gurp }\end{array}$ & $5 \mathrm{~b}$ & & $8420 \pm 110$ & $\begin{array}{l}723017597 \\
805558357\end{array}$ \\
\hline 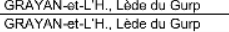 & & $\frac{\text { Ly-53322 }}{\text { Ly-5321 }}$ & $\frac{9200 \pm 70}{9180 \pm 90}$ & $\frac{8065538357}{8033: 8359}$ \\
\hline GRAYAN-E-LL'H., Lède du Gurp & & Beta-118447 & $9070+70$ & $7990 ; 8185$ \\
\hline GRAYAN-ot-L'H., Lòdo du Gurp & $11 \mathrm{c}$ & Ly-5325 & $8760 \pm 100$ & 754997978 \\
\hline GRAYAN-t-L'H., Lède du Gurp & US 411 & Beta-118446 & $8620 \pm 60$ & $7520 / 7715$ ou $7815 / 7870$ \\
\hline GRAYAN-at-L'H., Lède du Gurp & Basebrolé & Ly-7969 & $8600 \pm 55$ & $7511 / 7834$ \\
\hline GRAYAN-et-L'H., Lède du Gurp & US 113b & Beta-118450 & $8540 \pm 70$ & 746517635 \\
\hline GRAYAN-1-L'H., Lède du Gurp & $11 \mathrm{~d}$ & $L y-6048$ & $8360 \pm 60$ & 7255,7508 \\
\hline GRAYAN-et-L'H.,. Lède du Gurp & brallè & Ly-6043 & $8125 \pm 70$ & 699177310 \\
\hline GRAYAN-t-L'H., Lède du Gurp & & Gif- 5465 & $7870 \pm 150$ & \\
\hline GRAYAN-at-L'H., Lìde du Gurp & US 413 & Bêta-118445 & $7620 \pm 60$ & $6365 / 6495$ \\
\hline 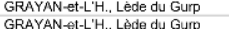 & US 410 & Béta-118451 & $7420 \pm 60$ & $6135: 6385$ \\
\hline 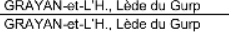 & & $\frac{L y-6045}{L y-646}$ & $7360 \pm 85$ & $\frac{6010 ; 6366}{5954 ; 6421}$ \\
\hline $\begin{array}{l}\text { GRAAAN-2-LL.L.Lede du GuP } \\
\text { LENTILAC-LAUZES, Fonttaures }\end{array}$ & $\frac{10}{6 \mathrm{~b}}$ & $\frac{L y-6046}{\text { Ly-4449 }}$ & $\begin{array}{l}7350 \pm 130 \\
9650 \pm 130\end{array}$ & 84950796060 \\
\hline LENTILLAC-LAUZES. Fonttauràs & $5 b$ & Ly-4448 & $9140 \pm 160$ & 7891,8593 \\
\hline Les Usclades & & Gif-8744 & $8220 \pm 70$ & $7255 / 7310$ \\
\hline MIERS, les Fieux & D3 base & Gif -1807 & $9450 \pm 190$ & $8128: 9009$ \\
\hline MIERS, les Fieux & plancher & Gif-4281 & $9060 \pm 190$ & $7589: 8500$ \\
\hline MILLAU, la Poujade & & Gif-3631 & $8990 \pm 200$ & $7750 / 8180$ \\
\hline MILLAU, Ia Poujade & $10 \mathrm{~B} / \mathrm{C}$ & MC-1240 & $8910 \pm 145$ & $7770: 8060$ \\
\hline MILLAU, Ia Poujade & $10 \mathrm{c}$ & Gif-3418 & $8710 \pm 190$ & 7510,7980 \\
\hline MOSTUEJJULS, LES Salzets & $c$ & Gifi-443 & $8770 \pm 200$ & $7423 / 8191$ \\
\hline REILHAC, grotte du Sanglier & 6 & Ly 7792 & $8075 \pm 75$ & 670817249 \\
\hline REILHAC, grotte du Sanglier & 6 & Ly-77933 & $8065 \pm 80$ & 66827244 \\
\hline $\begin{array}{l}\text { REILILAC, grotte du Sanglier } \\
\text { REIHAC, grotte du Sanglier }\end{array}$ & 6 base & Ly-6162 & $7943 \pm 76$ & $6396 / 7015$ \\
\hline $\begin{array}{l}\text { REIHACAC, grotte du Sanglier } \\
\text { REILHAC, grotte du Sanglier }\end{array}$ & $5 b$ & Ly-5687 & $7753 \pm 235$ & $6115 / 7241$ \\
\hline $\begin{array}{l}\text { REIIHAC, rrotte du Sanglier } \\
\text { ROUFFIGNAC }\end{array}$ & $\frac{5 b}{5 b}$ & $\frac{L y-6510}{G N-5514}$ & $\begin{array}{l}7557 \pm 104 \\
9150+90\end{array}$ & $\frac{6143 ; 6542}{8019: 356}$ \\
\hline ROUFFIGNAC & $4 c$ & $\begin{array}{ll}G N-5314 \\
G N-2880\end{array}$ & $\begin{array}{l}9150 \pm \pm 0 \\
8995 \pm 105\end{array}$ & $7877 / 8256$ \\
\hline ROUFFIGNAC & $5 a$ & GiN-5513 & $8750 \pm 75$ & 754777972 \\
\hline ROUFFIGNAC & $4 \mathrm{~b}$ & GrN-2895 & $8590 \pm 100$ & $7497 / 7701$ ou $7436 / 7908$ \\
\hline ROUFFIGNAC & $4 a$ & $G+N-2$ & $8370 \pm 100$ & $7283 / 7504$ ou $7194 / 7546$ \\
\hline ROUFFIGN & 3 base & GiN-2889 & $7800 \pm 50$ & $6464: 6706$ \\
\hline SAUVETERRE, Rac Allan & $\mathrm{T} 1$ & Lyy-4545 & $8160 \pm 90$ & $6604 / 7505$ \\
\hline SAUVETERRE, Roc Allan & & Ly-4931 & $7625 \pm 80$ & $5650 ; 6470$ \\
\hline St-CERNIN-de-LARCHE, la Doue & $2 \mathrm{~b}$ & Ly-2819 & $9260 \pm 200$ & $8060 ; 8520$ \\
\hline St-CERNIN-do-LARCHE, la Doue & $2 \mathrm{~b}$ & Ly-2820 & $8980 \pm 210$ & $7730: 8100$ \\
\hline St-CERNIN-de-LARCHE, la Doue & & Ly-2834 & $8880 \pm 160$ & $7850: 8040$ \\
\hline ti-CERNNIN-Ae-LARCHE, la Doue & $\frac{2 a}{2 a}$ & $\frac{L y-2821}{L y-233}$ & $\frac{8860 \pm 210}{8750+150}$ & $\frac{759008080}{75501980}$ \\
\hline THCERANA-de-LARCHE, la Doue & $3 c$ & $\frac{L y-2233}{L y-5273}$ & $\begin{array}{l}8750 \pm 150 \\
8625+80\end{array}$ & 751017830 \\
\hline & & & $8625 \pm 80$ & \\
\hline
\end{tabular}


Figure 11 - Datations radiocarbone du Mésolithique du sud-ouest de la France (d'après RoussotLarroque 2009).

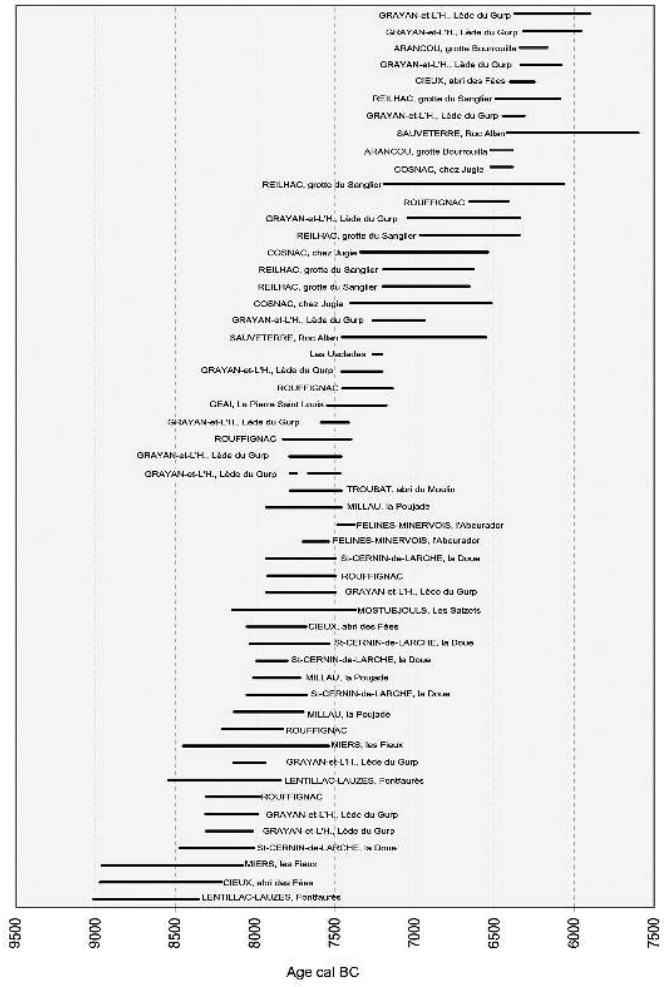

\section{BIBLIOGRAPHIE}

ADOVASIO J.M., SOFFER O., HYLAND D.C., KLIMA B. et SVOBODA J. 1999 - Perishable technologies and the genesis of the eastern Gravettian. Anthropologie (Brno), 36, $\mathrm{n}^{\circ} 1 / 2$, p. 43-68.

BRUSLE J. et QUIGNARD J-P. 2001 - Biologie des poissons d'eau douce européens. Collection Aquaculture-Pisciculture dirigée par J. Arrignon. Londres-Paris-New York : Editions TEC et DOC, 623 p., ill.

CAVA ALMUZARA A.C., ALDAY RUIZ A. et TARRI-O A. 2007-2008 - La circulación de materias primas líticas en la transición Mesolítico /Neolítico Antiguo en el País Vasco. Los abrigos de Mendandia, Kanpanoste y Aizpea, Veleia : Homenaje a Ignacio Barandiarán Maestu, n 24-25, tome I, p. 581-609, (Revista de Prehistoria, Historia antigua, Arqueología y Filología clásicas, Vitoria-Gasteiz).

CHAUCHAT Cl. (dir.), FONTUGNE M., HATTE C., DACHARY M. BONNISSENT D., CHAUVIÈRE F.-X., ROUSSOT A., FRITZ C., FOSSE Ph., EASTHAM A., MARTIN H., LE GALL O. et GAMBIER D. 1999 L'habitat magdalénien de la grotte du Bourrouilla à Arancou (Pyrénées-Atlantiques). Gallia Préhistoire, 41, p. 1-151.

CHESNAUX L. 2008 - Des microlithes sauveterriens, témoins de l'armement des derniers chasseurs-cueilleurs dans les Alpes du Nord. In : J.-M Pétillon., M.-H. Dias-Meirinho, P. Cattelain, 
M. Honegger, C. Normand et N. Valdeyron (coord.), Recherches sur les armatures de projectiles du Paléolithique supérieur au Néolithique. Actes du colloque C83, XVe congrès de l'UISPP, Lisbonne 2006. Palethnologie, 1, p. 139-153.

DACHARY M. (dir), CHAUVIÈRE F.-X., COSTAMAGNO S., EASTHAM A., FERRIER C., HENRYGAMBIER D. et VANHAEREN M. 2003 - Grotte de Bourrouilla (Arancou, Pyrénées-Atlantiques), Rapport intermédiaire de fouille programmée tri-annuelle : Deuxième campagne du 1er au 27 septembre 2003, Exemplaire multigraphié, SRA Aquitaine, 93 p.

DACHARY M. (dir), AURIÈRE L., CHAUVIÈRE F.-X., COSTAMAGNO S., EASTHAM A., FRITZ C., MALLYE J.-B., MERLET J.-Cl., MIQUÉOU M., PLASSARD F. et VANHAEREN M. 2010 - Grotte de Bourrouilla. Commune d'Arancou, Pyrénées-Atlantiques (64). DFS de la fouille programmée 2010, Exemplaire multigraphié, SRA Aquitaine, $165 \mathrm{p}$.

DACHARY M. (dir), AURIÈRE L., CHAUVIÈRE F.-X., COSTAMAGNO S., DRUCKER D., EASTHAM A., FERRIER C., FRITZ C., HENRY-GAMBIER D., LE GALL O., MERLET J.-Cl., MIQUÉOU M., PLASSARD F. et VANHAEREN M. 2012 - Grotte de Bourrouilla. Commune d'Arancou, Pyrénées-Atlantiques (64). Rapport intermédiaire de fouille programmée tri-annuelle : Première campagne du 29 août au 29 septembre 2012, Exemplaire multigraphié, SRA Aquitaine, 244 p.

DACHARY M. 1993 - Étude d'une collection lithique du Paléolithique supérieur. Gisement de Bourrouilla, commune d'Arancou, Département des Pyrénées Atlantiques. Nantes : Université de Nantes, 2 t., 136 p., 51 pl. Mémoire de maîtrise.

DETRAIN L., TURQ A. et VIGIER S. 2006 - Une occupation sauveterrienne en abri-sous-roche : le Roc Allan (Sauveterre-la-Lémance, Lot-et-Garonne). Paysages et peuplements. Aspects culturels et chronologiques en France méridionale. Actualité de la recherche. Périgueux, Coédition ADRAHP et PSO, p. 445-454.

DOMINGO MARTINEZ R. 2005 - La funcionalidad de los microlitos geométrico : bases experimentales para su estudio, Monografías arqueológicas $n^{\circ} 42$, Departamento de ciencias de la Antigüedad Universidad de Zaragoza, Zaragoza.

FISCHER A., WEMMING-HANSEN P. et RASMUSSEN P. 1984 - Macro and micro wear traces on lithic projectile points : experimental results and prehistoric examples, Journal of Danish archaeology, 3, p. 19-46.

G.E.E.M. 1969 - Epipaléolithique-Mésolithique. Les armatures géométriques-2, Bulletin de la Société Préhistorique Française, Etudes et Travaux, 66, p. 355-366.

G.E.E.M. 1972 - Epipaléolithique-Mésolithique. Les armatures non géométriques-1, Bulletin de la Société Préhistorique Française, Etudes et Travaux, 69, p. 364-375.

G.E.E.M. 1975 - Epipaléolithique-Mésolithique. L'outillage du fonds commun-1, Bulletin de la Société Préhistorique Française, Etudes et Travaux, 72, p. 319-332.

GHESQUIÈRE E. et MARCHAND G. 2010 - Le Mésolithique en France, archéologie des derniers chasseurs-cueilleurs. Paris : La Découverte, 178 p.

KETTLE J., HEINRICH D., BARRET J.H., BENECKE N. et LOCKER A. 2008 - Past-distributions of the European freshwater eel from archaeological and palaeontological evidence. Quaternary Science Reviews, 27, p. 1309-1334.

LE GALL O. 1982 - Les poissons de quelques gisements préhistoriques du Sud-Ouest de la France. Etude ostéologique. Intérêt paléoécologique et palethnologique. Bordeaux : Université de Bordeaux I, 1 t., 177 p., 103 pl. Thèse de doctorat en Géologie du Quaternaire et Préhistoire. $n^{\circ} 1760$. 
LE GALL O. 1984 - L'ichtyofaune d'eau douce dans les sites préhistoriques. Ostéologie- Paléoécologie- Palethnologie. Les Cahiers du Quaternaire, n VIII, Ed. CNRS, 196 p., 55 fig.

LE GALL O. 1995 - Étude des poissons. In : M. R. SERONIE-VIVIEN : La grotte de Pégourié, Caniacdu-Causse (Lot). Préhistoire Quercinoise - Supplément n², p. 149-154.

LE GALL O. 1999 - Ichtyophagie et pêches préhistoriques. Quelques données de l'Europe occidentale. Bordeaux : Université de Bordeaux I, 1 t., 473 p., 86 pl., 35 tab., 39 fig. Thèse présentée pour obtenir le grade de Docteur d'Etat ès Sciences.

LE GALL 0. 2003 - La squelettochronologie appliquée aux poissons. Une méthode de reconnaissance des saisons de capture. Préhistoire du Sud-Ouest, 10, n 1, p. 9-24.

LE GALL 0. 2008 - Les poissons des eaux douces pléistocènes sont-ils des indicateurs paléoclimatiques ? Une approche élargie à l'Europe. In : P. Béarez, S. Grouard et B. Clavel (Ed.), Archéologie du Poisson. Exploitations et impacts, transformations et usages, Paléoenvironnements. 30 ans d'Archéo-ichtyologie au CNRS. Hommage aux travaux de Jean Desse et Nathalie Desse-Berset. 14th ICAZ Fisch Remains Working Group Meeting. XXVIIIémes Rencontres Internationales d'Archéologie et d'Histoire d'Antibes. Editions APDCA, Antibes 2008, p. 311-326.

LE GALL 0. 2010 - Influences des glaciaires-interglaciaires sur les ichtyofaunes des eaux douces européennes. Actes du colloque international Q6, AFEQ, INQUA. Montpellier, 25-28 Février 2008. Quaternaire, t. 21, (1), p. 203-214.

LE GALL 0. 2011 - Origines des restes de poissons d'eaux douces dans les sites paléolithiques : un point sur la question. In : Laroulandie V., Mallye J.-B., Denys Ch. (eds), Taphonomie des petits vertébrés : référentiels et transferts aux fossiles : actes de la table ronde du RTP Taphonomie, Talence 20-21 octobre 2009, Oxford : Archaeopress, 2011, p. 153-166 (BAR International Series, 2269).

LE GALL O., LENOIR M. et BELBEOC'H G. 2006 - Le galet gravé de la Honteyre (Tuzan, Gironde). Préhistoire du Sud-Ouest, 12, 2, p. 175-188.

MALLYE J.-B. 2007 - Les restes de Blaireau en contexte archéologique : Taphonomie, Archéozoologie et éléments de discussion des séquences préhistoriques. Bordeaux : Université de Bordeaux I, Thèse de doctorat.

MALLYE J.-B. 2011 - Réflexion sur le dépouillement des petits carnivores en contexte archéologique : Apport de l'expérimentation. Archaeofauna, 20, p. 7-25.

MARCHAND G. 1999 -Typologie lithique appliquée au Mésolithique et au Néolithique de l'Ouest de la France. In : La néolithisation de l'Ouest de la France : caractérisation des industries lithiques, BAR, International Series, 748, $487 \mathrm{p}$.

MARCHAND G. et MANEN C. 2006 - Le rôle du Néolithique ancien méditerranéen dans la néolithisation de l'Europe atlantique. In : Paysages et peuplements, Actes des 6e Rencontres Méridionales de Préhistoire Récente (Périgueux, 2004), Périgueux, p. 213-232.

MERLET J.-Cl. et MIQUÉOU M. 2009 -- Une approche du Mésolithique des Landes de Gascogne : Gaillèbes-2 à Sabres (Landes), Archéologie des Pyrénées occidentales et des Landes, 28, p. 7-17.

MIQUÉOU M. 2012 - Les armatures en double biseau : indices d'interactions transpyrénéennes dans le processus de néolithisation de l'Aquitaine, Archéologie des Pyrénées occidentales et des Landes, 29, p. 53-65. 
PERRIN T., MARCHAND G., ALLARD P., BINDER D., COLLINA C., GARCIA-PUCHOL O., VALDEYRON

N. 2009 - Le second Mésolithique d'Europe occidentale : origines et gradient chronologique, Annales de la Fondation Fyssen, 24, p. 160-177.

ROUSSOT-LARROQUE J. 2005 - Le Sud-Ouest aquitain entre sud et ouest, du VIIIe au Ve millénaire avant notre ère. In : Territoires, déplacements, mobilité, échanges durant la Préhistoire, Actes du 126e congrès national des sociétés historiques et scientifiques (Toulouse, 2001), Paris, CTHS, p. 471-497.

ROUSSOT-LARROQUE J. 2009 - Les complexes géométriques en Aquitaine. In : P. Utrilla Miranda et L. Ramirez Sanchez (Eds.), El Mesolítico Geométrico en la Península Ibérica, Actes du colloque de Jaca (2008), Zaragoza : Monographías Arquelógicas, 44, p. 279-306.

ROUSSOT-LARROQUE J. 2011 - Les derniers grands chasseurs des landes d'Aquitaine. In : Merlet J.-Cl. et Bost J.-P. (dir.) : De la lagune à l'airial, Le peuplement de la Grande Lande, Aquitania, suppl. 24, APOL, Hors série 5, p. 77-116.

SÉRONIE-VIVIEN M.-R. (dir.) 2001 - La grotte du Sanglier à Reilhac (Lot). Du Magdalénien au Néolithique ancien. Préhistoire du Sud-Ouest, suppl. 4, 182 p.

SOFFER O., ADOVASIO J.M., ILLINGWORTH J.S., AMIRKHANOV H.A., PRASLOV N.D. et STREET M. 2000 - Palaeolithic perishables made permanent. Antiquity, 74, p. 812-821.

SPILLMANN Ch. J. 1961 - Poissons d'eau douce. Faune de France n 65. Paris : Paul Lechevallier, 303 p., ill.

SZMIDT C., LAROULANDIE V., DACHARY M., LANGLAIS M. et COSTAMAGNO S. 2009 - Harfang, Renne et Cerf : nouvelles dates ${ }^{14} \mathrm{C}$ par SMA du Magdalénien supérieur du Bassin aquitain au Morin (Gironde) et Bourrouilla (Pyrénées-Atlantiques), Bulletin de la Société Préhistorique Française, 106, $\mathrm{n}^{\circ}$ 3, p. 583-587.

VALDEYRON N. 2000 - Géographie culturelle du Mésolithique recent/final dans le sud-ouest de la France, Sociétés et espaces, Actes des 3e Rencontres Méridionales de Préhistoire Récente, Archives d'Ecologie Préhistorique, Toulouse, p. 23-34.

VALDEYRON N., BOSC-ZANARDO B., BRIAND T. 2009 - Évolutions des armatures de pierre et dynamiques culturelles durant le Mésolithique dans le sud-ouest de la France : l'exemple du Haut-Quercy (Lot, France). In : Recherches sur les armatures de projectiles du Paléolithique supérieur au Néolithique, Actes du colloque C83, XVe congrès de l'UISPP, Lisbonne, 4-9 septembre 2006, Revue en ligne Palethnologie, n 1, p. 269-286.

\section{RÉSUMÉS}

Le Mésolithique de l'extrême sud-ouest de la France est aujourd'hui encore mal connu puisque que peu de gisements ont fourni des données fiables entre le sud des Landes et la frontière espagnole. La grotte de Bourrouilla à Arancou (Pyrénées-Atlantiques) apporte de précieuses données grâce à la découverte de témoins d'occupations associant vestiges lithiques et faune (mammifères, poissons et oiseaux).

Trois locus, offrant des degrés de conservation différents, documentent inégalement un Premier Mésolithique avancé, un Second Mésolithique à trapèzes et un Néolithique ancien à segments du Bétey. Les études archéozoologiques permettent de percevoir l'exploitation de deux biotopes : la forêt et les cours d'eau. Le spectre de la faune chassée est dominé par le Cerf, le Chevreuil et le Sanglier, auxquels sont associés le Blaireau, la Martre et la Buse variable tandis que l'ichtyofaune est surtout représentée par les cyprinidés. Les données de saisonnalité convergent vers des 
occupations au début/milieu de la bonne saison. En outre, deux datations radiocarbone contribuent au débat sur la transition entre Premier et Second Mésolithique.

The Mesolithic of the extreme southwest of France is still poorly understood because there are few sites that provide reliable data between the region of the southern Landes and the Spanish border. Bourrouilla cave at Arancou (Pyrénées-Atlantiques) has revealed some interesting information, following the discovery of occupation levels with evidence of Mesolithic lithic artefacts and fauna (mammal, fish, and bird).

Three locations, producing material in differing states of preservation, have provided data about a late, early Mesolithic occupation, a later Mesolithic with trapezoid microliths and an early Neolithic with segments of Betey.

The archaeozoological studies allow us to assess the exploitation of two biotopes: the forest and the rivers. The spectrum of hunted fauna is dominated by red deer, roe deer and wild boar, in association with badger, marten and common buzzard, while the fish remains are mainly cyprinids. The data on the seasonal occupation of the site converges on a time at the beginning or middle of the good season. In addition, there are two radiocarbon dates relevant to a discussion about the transition between the early and later Mesolithic occupations

\section{INDEX}

Mots-clés : Mésolithique, Néolithique ancien, Pyrénées-Atlantiques, typo-technologie lithique, archéozoologie, saisonnalité, datation radiocarbone

Keywords : Mesolithic, early Neolithic, Pyrénées-Atlantiques, lithic typo-technology, archaeozoology, seasonality, radiocarbon dating

\section{AUTEURS}

\section{MORGANE DACHARY}

UMR 5608 - TRACES, Université Toulouse-2-le-Mirail - morgane-dachary@orange.fr

\section{JEAN-CLAUDE MERLET}

CRAL, 19 rue des moissons, FR-40180 Narrosse - merlet.jeanclaude@orange.fr

\section{MATHILDE MIQUÉOU}

CRAL, 19 rue des moissons, FR-40180 Narrosse.

\section{JEAN-BAPTISTE MALLYE}

Université Bordeaux 1, PACEA, UMR 5199, CNRS, FR-33405 Talence - b_mallye@yahoo.fr

\section{OLIVIER LE GALL}

Université Bordeaux 1, PACEA, UMR 5199, CNRS, FR-33405 Talence - o.le-gall@pacea.ubordeaux1.fr

\section{ANNE EASTHAM}

Dolau, Dwrbach, Fishguard, Pembrokeshire, Pays de Galles, Grande-Bretagne eastham146@btinternet.com 